\title{
A Supply and Demand Model of the College Admissions Problem
}

\section{Citation}

Chade, Hector, Gregory Lewis, and Lones Smith. 2009. A Supply and demand model of the college admissions problem. Faculty working paper, Department of Economics, Harvard University.

\section{Published Version}

http://www.economics.harvard.edu/faculty/lewis/files/cls2009_dec.pdf

\section{Permanent link}

http://nrs.harvard.edu/urn-3:HUL.InstRepos:4481401

\section{Terms of Use}

This article was downloaded from Harvard University's DASH repository, and is made available under the terms and conditions applicable to Open Access Policy Articles, as set forth at http:// nrs.harvard.edu/urn-3:HUL.InstRepos:dash.current.terms-of-use\#OAP

\section{Share Your Story}

The Harvard community has made this article openly available.

Please share how this access benefits you. Submit a story.

\section{Accessibility}




\title{
A Supply and Demand Model of the College Admissions Problem*
}

\author{
Hector Chade ${ }^{\dagger} \quad$ Gregory Lewis ${ }^{\ddagger} \quad$ Lones Smith $^{\S}$
}

First version: July 2003

This version: December 12, 2009

\begin{abstract}
We develop the first Bayesian model of decentralized college admissions, with heterogeneous students, costly portfolio applications, and noisy college evaluations. Students face a nontrivial portfolio choice, and colleges choose admissions standards that act like market-clearing prices.

We derive the two college model equilibrium, deriving a "law of demand". Surprisingly, the worse college might impose higher standards, and weaker students sometimes apply more aggressively. The lesser college impacts its rival through student portfolio reallocation. Also, the weaker college counters affirmative action at its rival with a discriminatory admissions policy, and may poach students from its rival using early admissions.
\end{abstract}

*An earlier version was called "The College Admissions Problem with Uncertainty." Greg and Lones are grateful for the financial support of the National Science Foundation. We have benefited from seminars at BU, UCLA, Georgetown, HBS, the 2006 Two-Sided Matching Conference (Bonn), 2006 SED (Vancouver), 2006 Latin American Econometric Society Meetings (Mexico City), and 2007 American Econometric Society Meetings (New Orleans), Iowa State, Harvard/MIT, the 2009 Atlanta NBER Conference, and Concordia. Parag Pathak and Philipp Kircher provided useful discussions of our paper. We are also grateful to John Bound and Brad Hershbein for providing us with student college applications data.

${ }^{\dagger}$ Arizona State University, Department of Economics, Tempe, AZ 85287-3806.

${ }^{\ddagger}$ Harvard University, Department of Economics, Cambridge, MA 02138.

${ }^{\S}$ University of Michigan, Economics Department, Ann Arbor, MI 48109-1220. 


\section{Contents}

1 Introduction $\quad 1$

2 The Model $\quad 4$

3 Equilibrium Analysis for Students $\quad 6$

3.1 The Student Optimization Problem . . . . . . . . . . . . . . . 6

3.2 Admission Chances and Student Calibers . . . . . . . . . . . . . . 8

4 Equilibrium Analysis for Colleges $\quad 10$

4.1 A Supply and Demand Approach . . . . . . . . . . . . . . . . . . . 10

4.2 Changing College Sizes and Application Costs . . . . . . . . . . . . . . 13

5 Do Colleges and Students Sort in Equilibrium? $\quad 15$

6 The Spillover Effects of Affirmative Action $\quad 17$

$\begin{array}{lll}7 & \text { Early Admissions } & 20\end{array}$

8 Concluding Remarks $\quad 23$

A Appendix: Proofs $\quad 24$

A.1 Colleges Optimally Employ Admissions Thresholds . . . . . . . . . . . . 24

A.2 Simultaneous versus Sequential Timing . . . . . . . . . . . . . . . 25

A.3 Acceptance Function and Signals . . . . . . . . . . . . . . . . . . . . . 25

A.4 Acceptance Function Shape: Proof of Theorem 1 . . . . . . . . . . . . . 26

A.5 Monotone Student Strategies . . . . . . . . . . . . . . . . . . . . . . 27

A.6 The Law of Demand . . . . . . . . . . . . . . . . . . . . . . . . . . . . . 29

A.7 Existence of a Stable Equilibrium . . . . . . . . . . . . . . . . . . . 29

A.8 Sorting Equilibrium Implies Stochastic Dominance of Types . . . . . . . 31

A.9 Changing Application Costs . . . . . . . . . . . . . . . . . . . . . . 32

A.10 Sorting and Non-Sorting: Proof of Theorem 2 . . . . . . . . . . . . . . . 34

A.11 Affirmative Action: Negligible Feedback Effects . . . . . . . . . . . . . . 37

A.12 Student Poaching: Proof of Theorem $4 \ldots \ldots$. . . . . . . . . . . . . . 38 


\section{Introduction}

The college admissions process has lately been the object of much scrutiny, both from academics and in the popular press. This interest owes in part to the strategic nature of college admissions. Schools competitively set admissions standards to attract the best students, and students in turn respond most judiciously in making their application decisions. This paper examines the joint behavior of students and colleges in equilibrium.

We develop and explore an equilibrium model of the college admissions process, with decentralized matching of a heterogeneous population of students, and two colleges one better and one worse, respectively, called 1 and 2 . The model captures two previously unexplored frictions relevant in the "real-world". First, college applications are costly and so students must solve a nontrivial portfolio choice problem. Second, colleges seek to fill their capacity with the best students possible, but only observe a noisy signal of each student's caliber. This tandem of costly and yet noisy applications feeds the intriguing conflict at the heart of the student choice problem: Gamble on Harvard, settle for Michigan, or apply to both. Meanwhile, college enrollments are interdependent, both because the student portfolios depend on the joint college admissions standards, and because students accepted at the better college will not attend the lesser one even if accepted. This asymmetric interdependence leads to an array of surprising results.

Central to our paper is a theorem characterizing how student acceptance chances at the colleges vary with student caliber. Building on our Bayesian foundation, we find that as a student's caliber rises, the ratio of his admission chances at college 1 to college 2 rises monotonically. This property, combined with student optimization, has strong and testable implications for how portfolio choices across students are related. Next, we consider the game induced among colleges by this optimal student behavior. We show how to analyze equilibrium through the lens of supply and demand: When a college raises its standards, its enrollment falls both because fewer students make the cut the standards effect - and as fewer will apply ex ante — the portfolio effect. Treating admissions standards as prices, these effects reinforce each other. In equilibrium, we uncover a "law of demand", in which a college's enrollment falls in its standard. Also, the portfolio effect increases the elasticity of this demand curve.

Analogous to Bertrand competition with differentiated products, colleges choose admissions standards to fill their desired enrollment, taking rival standards and the student portfolios as given. An equilibrium occurs when both markets clear and students behave optimally. The model frictions yield some novel comparative statics. For instance, the 
admissions standards at both colleges fall if college 2 raises its capacity, while lower application costs at either school increase the admissions standards at the better college. So UCLA cannot ignore admissions activity at the weaker cross-town rival USC.

The second part of the paper attacks the question of whether sorting occurs in equilibrium: Do the better students apply more "aggressively" ? ${ }^{1}$ Does the better college impose higher admissions standards? Surprisingly, we show that neither form of sorting need hold in general. For example, if both colleges set equal standards, the worse college will still attract insurance applications from middling students. So given equal standards that clear the market at college 1, if college 2 is small so that projected enrollment exceeds capacity, then by our "law of demand" it must raise its standards above college 1 in equilibrium. Conversely, we prove that all equilibria involve sorting if the colleges differ sufficiently in quality and the higher ranked school is not too small.

To close the paper, we enrich the strategy space in our model to provide a unified analysis of two seemingly unrelated topics: early admissions and affirmative action. We let colleges offer different standards to observably different students — such as early applicants or members of a target class of students. As occurs with third degree price discrimination, we show that colleges act in both cases to equate the "shadow value" of different groups. For instance, the worse college can use early admissions to poach students from the better one. The reason is that an early acceptance there lowers a student's marginal benefit of a regular application to the better college.

We discover that when the better college initiates an affirmative action policy favoring one group, the weaker college responds by penalizing that group. We broadly interpret affirmative action to include favoring disadvantaged minorities, as well as the differential preferential treatment for athletes, and state resident preferences or mandates on public colleges. Since $90 \%$ of UCLA students are state residents, our result suggests that USC should raise the bar for in-state students. This occurs because of an "acceptance curse": a favored student enrolling at the weaker college may have been rejected by the better college despite affirmative action, and this is a bad signal of their caliber.

Our analysis highlights the role of frictions - costly and uncertain applications in college admissions. A natural question then is whether these frictions are important in practice. Certainly, application outcomes are far from guaranteed: Students face admissions rates well below $50 \%$ at the top colleges, and are routinely advised to construct

\footnotetext{
${ }^{1}$ Precisely, the best students apply just to college 1; weaker students insure by applying to both colleges; even weaker ones apply just to college 2; and finally, the weakest apply nowhere.
} 
thoughtful portfolios that include both "safety" and "stretch" schools. Yet one might wonder whether application costs - either in money or time - are sufficient to limit the number of applications, given the large marginal benefit to attending a better college.

The answer is yes. Data from the Higher Education Research Institute's freshman survey reveal that the modal number of college applications has been one since 1975 (see our supplementary appendix). The mean number of applications has risen over time, to 4.3 in 2006. This low level is consistent with a portfolio choice model, since the marginal benefit to an additional application falls geometrically. For example, an average student applying to identical average four-year colleges with $75 \%$ acceptance rate has a marginal benefit to her 5 th application scaled by $4^{-4}=1 / 256$; so that even if attending college yields surplus of $\$ 20000$, the marginal benefit of that application is only $\$ 59$. The trend in applications also accords with our model, which would predict more applications as application costs have fallen due to the Internet and the Common Application.

Modeling frictions is not only important to match stylized facts, but also overturns a number of conclusions reached in their absence. While sorting is trivial absent frictions, it proves elusive with frictions. This adds to the literature on decentralized frictional matching - e.g., Shimer and Smith (2000), Smith (2006), Chade (2006), and Anderson and Smith (2007). Our finding that weaker colleges can sustain higher standards in equilibrium is a cautionary tale to those who rank colleges by their admissions standards.

The student portfolio problem embedded in the model is a special case of the problem solved in Chade and Smith (2006). But the acceptance chances here are endogenous, since any one student's acceptance chance depends on the equilibrium college admission threshold. So our paper also contributes to the literature on equilibrium models with non-sequential search (e.g. Burdett and Judd (1983), Burdett, Shi, and Wright (2001), Albrecht, Gautier, and Vroman (2003) and Kircher and Galenianos (2006)).

The importance of college sorting has recently been documented in Hoxby (2009). Our affirmative action result turns on the acceptance curse (Chade 2006). Finally, early admissions serves as a poaching device in our setup. Both Lee (2009) and Avery and Levin (2009) have shown that early admissions can be useful in a world with preference heterogeneity, allowing students to signal their interest in attending a particular school (for an overview of early admissions, see Avery, Fairbanks, and Zeckhauser (2003)).

After introducing the model, we do the student and college equilibrium analyses, respectively. We then explore the sorting character of equilibria. We conclude with explorations of affirmative action and early admissions. Proofs are found in the appendix. 


\section{The Model}

A. An Overview. The paper introduces three key features - heterogeneous students, portfolio choices with unit application costs, and noisy evaluations by colleges. We impose little additional structure. For instance, we ignore the important and realistic consideration of heterogeneous student preferences over colleges.

A central feature of our analysis is modeling college portfolio applications. Student choice is trivial if it is costless, and in practice, such costs can be quite high. Indeed, the sole purpose of the Common Application is to lower the cost of multiple applications. ${ }^{2}$

Next, we assume noisy signals of student calibers. This informational friction creates uncertainty for students, and a Bayesian filtering problem for colleges. It captures the difficulty faced by market participants, with students choosing "safety schools" and "stretch schools", and colleges trying to infer the best students from noisy signals. Without noise, sorting would be trivial: Better students would apply and be admitted to better colleges, for their caliber would be correctly inferred and they would be accepted. As we will see, sorting is less easily achieved with both application costs and evaluation noise. Indeed, there is a richer role for student choice in this environment.

We also make two other key modeling choices. First, we assume just two colleges. This is done for the sake of tractability. We discuss the applicability of our insights for the much more challenging $n$-college problem en route. We also fix the capacity of the two colleges. This is most defensible in the short run, and so it is best to interpret our model as focusing on the "short run" analysis of college admissions. We explore the simultaneous game in which students apply to college, and colleges decide whom to admit. However, we later briefly explore the possibility of "early admissions".

A model with both common and idiosyncratic components for student evaluation is intractable. We can solve both extreme cases with perfectly correlated and conditionally i.i.d. signals. ${ }^{3}$ But we restrict the analysis to the less trivial latter case. It is arguably more realistic too, since admission to a better college often does not guarantee admission to a lesser one. By contrast, perfect correlation would force this implication. Conditionally i.i.d. signals exactly captures the case where students are apprised of all variables (such as the ACT/SAT or their GPA) common to their applications before

\footnotetext{
${ }^{2}$ This general application form is used by almost 400 colleges, and simplifies college applications. It eliminates idiosyncratic college requirements, but retains separate college application fees.

${ }^{3}$ Proof available upon request. Nagypál (2004) analyzes the decision problem facing a student with perfectly correlated admissions.
} 
applying to college. Students are uncertain as to how these idiosyncratic elements such as college-specific essays and interviews will be evaluated, but believe that the resulting signals are conditionally independent across colleges.

B. The Model. There are two colleges 1 and 2 with capacities $\kappa_{1}$ and $\kappa_{2}$, and a unit mass of students with calibers $x$ whose distribution has a density $f(x)$ over $[0, \infty)$. We avoid trivialities, and assume that college capacity is insufficient for all students, as $\kappa_{1}+\kappa_{2}<1$. Each college application costs a student $c>0$. All students preferring college 1. Everyone receives payoff 1 for attending college $1, u \in(0,1)$ for college 2 , and zero payoff for not attending college. To avoid trivialities, we later bound application costs above. Students maximize expected college payoff less application costs. College payoff equals the average enrolled student caliber times the measure of students enrolled.

Students know their caliber, and colleges do not. Colleges 1 and 2 each just observes a noisy conditionally independent signal of each applicant's caliber. In particular, they do not know where else students have applied. Signals $\sigma$ are drawn from a conditional density function $g(\sigma \mid x)$ on a subinterval of $\mathbb{R}$, with $\operatorname{cdf} G(\sigma \mid x)$. We assume that $g(\sigma \mid x)$ is continuous and obeys the strict monotone likelihood ratio property (MLRP). So $g(\tau \mid x) / g(\sigma \mid x)$ is increasing in the student's type $x$ for all signals $\tau>\sigma$.

Students apply simultaneously to either, both, or neither college, choosing for each caliber $x$, a college application menu $S(x)$ in $\{\varnothing,\{1\},\{2\},\{1,2\}\}$. Colleges choose the set of acceptable students signals. They intuitively should use admission standards to maximize their objective functions, so that college $i$ admits students above a threshold signal $\underline{\sigma}_{i}$. Appendix A.1 proves this given the MLRP property - despite an acceptance curse that college 2 faces (as it may accept a reject of college 1 ).

For a fixed admission standard, we want to ensure that very high quality students are almost never rejected, and very poor students are almost always rejected. For this, we assume that for a fixed signal $\sigma$, we have $G(\sigma \mid x) \rightarrow 0$ as $x \rightarrow \infty$ and $G(\sigma \mid x) \rightarrow 1$ as $x \rightarrow 0$. For instance, exponentially distributed signals have this property $G(\sigma \mid x)=$ $1-e^{-\sigma / x}$. More generally, this obtains for signals drawn from any "location family", in which the conditional cdf of signals $\sigma$ is given by $G((\sigma-x) / \mu)$, for any smooth cdf $G$ and $\mu>0$ - e.g. normal, logistic, Cauchy, or uniformly distributed signals. The strict MLRP then holds if the density is log-concave, so that $\log G^{\prime}$ is strictly concave.

C. Equilibrium. We consider a simultaneous move game by colleges and students. This yields the same equilibrium prediction as when students move first, as they are 
atomless. ${ }^{4}$ A Nash equilibrium is a triple $\left(S^{*}(\cdot), \underline{\sigma}_{1}^{*}, \underline{\sigma}_{2}^{*}\right)$ such that:

(a) Given $\left(\underline{\sigma}_{1}^{*}, \underline{\sigma}_{2}^{*}\right), S^{*}(x)$ is an optimal college application portfolio for each $x$,

(b) Given $\left(S^{*}(\cdot), \underline{\sigma}_{j}^{*}\right)$, college $i$ 's payoff is maximized by admissions standard $\underline{\sigma}_{i}^{*}$.

In a sorting equilibrium, colleges' and students' strategies are monotone. This means that the better college is more selective $\left(\underline{\sigma}_{1}^{*}>\underline{\sigma}_{2}^{*}\right)$ and higher caliber students are increasingly aggressive in their portfolio choice: The weakest apply nowhere; better students apply to the "easier" college 2; even better ones "gamble" by applying also to college 1; the next tier up shoot an "insurance" application to college 2; finally, the top students confidently just apply to college 1. Monotone strategies ensure the intuitive result that the distribution of student calibers at college 1 first-order stochastically dominates that of college 2 (see Claim 3 in Appendix A.8), so that all top student quantiles are larger at college 1 . This is the most compelling notion of student sorting in our environment with noise (Chade 2006).

Our concern with a sorting equilibrium may be motivated on efficiency grounds. If there are complementarities between student caliber and college quality, so that welfare is maximized by assigning the best students to the best colleges, any decentralized matching system must necessarily satisfy sorting to be (constrained) efficient. Since formalizing this idea would add notation and offer little additional insight, we have abstracted from these normative issues and focused on the positive analysis of the model.

\section{Equilibrium Analysis for Students}

\subsection{The Student Optimization Problem}

We begin by solving for the optimal college application set for a given pair of admission chances at the two colleges. ${ }^{5}$ Consider the portfolio choice problem for a student facing the admission chances $0 \leq \alpha_{1}, \alpha_{2} \leq 1$. The expected payoff of applying to both colleges is $\alpha_{1}+\left(1-\alpha_{1}\right) \alpha_{2} u$. The marginal benefit $M B_{i j}$ of adding college $i$ to a portfolio of

\footnotetext{
${ }^{4}$ See Appendix A.2. Alternatively, colleges could move first, committing to an admission standard. This is arguably not the case, but regardless, it too yields the same equilibria until we study affirmative action and early admissions (proof omitted). In the interests of a unified treatment throughout the paper, we proceed in the simultaneous move world.

${ }^{5}$ Chade and Smith (2006) provide an algorithm that would be useful in the $n$-college case. In our two college world, their analysis is trivial, and is not needed here.
} 

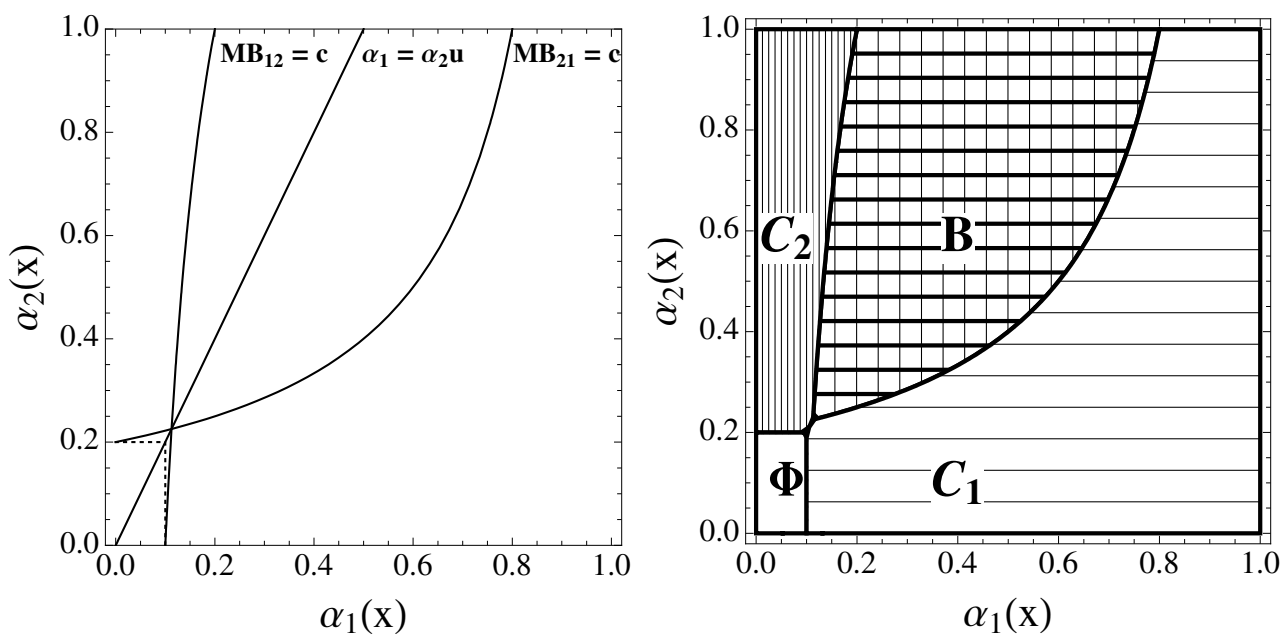

Figure 1: Optimal Decision Regions. The left panel depicts $(i)$ a dashed box, inside which applying anywhere is dominated; $(i i)$ the indifference line for solo applications to colleges 1 and 2; and (iii) the marginal benefit curves $M B_{12}=c$ and $M B_{21}=c$ for adding colleges 1 or 2 . The right panel shows the optimal application regions. A student in the blank region $\Phi$ does not apply to college. He applies to college 2 only in the vertical shaded region $C_{2}$; to both colleges in the hashed region $B$, and to college 1 only in the horizontal shaded region $C_{1}$.

college $j$ is then:

$$
\begin{aligned}
M B_{21} & \equiv\left[\alpha_{1}+\left(1-\alpha_{1}\right) \alpha_{2} u\right]-\alpha_{1}=\left(1-\alpha_{1}\right) \alpha_{2} u \\
M B_{12} & \equiv\left[\alpha_{1}+\left(1-\alpha_{1}\right) \alpha_{2} u\right]-\alpha_{2} u=\alpha_{1}\left(1-\alpha_{2} u\right)
\end{aligned}
$$

The optimal application strategy is then given by the following rule:

(a) Apply nowhere if costs are prohibitive: $c>\alpha_{1}$ and $c>\alpha_{2} u$.

(b) Apply just to college 1 , if it beats applying just to college $2\left(\alpha_{1} \geq \alpha_{2} u\right.$ ), and nowhere $\left(\alpha_{1} \geq c\right)$, and to both colleges $\left(M B_{21}<c\right.$, i.e. adding college 2 is worse).

(c) Apply just to college 2, if it beats applying just to college $1\left(\alpha_{2} u \geq \alpha_{1}\right)$, and nowhere $\left(\alpha_{2} u \geq c\right)$, and to both colleges $\left(M B_{12}<c\right.$, i.e. adding college 1 is worse).

(d) Apply to both colleges if this beats applying just to college $1\left(M B_{21} \geq c\right)$, and just to college $2\left(M B_{12} \geq c\right)$, for then, these solo application options respectively beat applying to nowhere, as $\alpha_{1}>M B_{12} \geq c$ and $\alpha_{2} u>M B_{21} \geq c$ by (1)-(2).

This optimization problem admits an illuminating and rigorous graphical analysis. The left panel of Figure 1 depicts three critical curves: $M B_{21}=c, M B_{12}=c, \alpha_{1}=u \alpha_{2}$. 
From (1) and (2), we see that all three curves share a crossing point, since $M B_{21}=M B_{12}$, when $\alpha_{1}=u \alpha_{2}$. Since $M B_{12}=c(1-c)<c$, this crossing point lies above and right of the point $\alpha_{1}=u \alpha_{2}=c$, below which applying anywhere is dominated.

Throughout the paper, we assume that $c<u(1-u)$. For if not, then the curves $M B_{21}=c$ and $M B_{12}=c$ cross a second time inside the unit square. ${ }^{6}$ The analysis then trivializes because multiple college applications need not occur.

Cases $(a)-(d)$ partition the unit square into regions of $\left(\alpha_{1}, \alpha_{2}\right)$ that correspond to each portfolio choice, suggestively denoted $\Phi, C_{2}, B, C_{1}$. These regions are shaded in the right panel of Figure 1. This picture summarizes the optimal portfolio choice of a student with arbitrary admissions chances $\left(\alpha_{1}, \alpha_{2}\right)$.

For an alternative insight into the student optimization, we could apply the marginal improvement algorithm of Chade and Smith (2006). There, a student first decides whether she should apply anywhere. If so, she asks which college is the best singleton. In Figure 1 at the left, college 1 is best right of the line $\alpha_{1}=u \alpha_{2}$, and college 2 is best left of it. Next, she asks whether she should apply anywhere else. Intuitively, there are two distinct reasons for applying to both colleges that we can now parse: Either college 1 is a "stretch" school (as a gamble) — namely, added second as a lower-chance higher payoff option - or college 2 is a "safety school", added second for insurance. In Figure 1, these are the parts of region $B$ above and below the line $\alpha_{1}=u \alpha_{2}$, respectively.

\subsection{Admission Chances and Student Calibers}

For known acceptance chances, we have seen that the optimization is rather straightforward. But we wish to predict the portfolio decisions of a heterogeneous continuum of students whose acceptance chances are endogenous. To this end, we now derive a mapping from student types to student application portfolios. Let us fix the thresholds $\underline{\sigma}_{1}$ and $\underline{\sigma}_{2}$ set by college 1 and college 2. Student $x$ 's acceptance chance at college $i$ is now given by $\alpha_{i}(x) \equiv 1-G\left(\underline{\sigma}_{i} \mid x\right)$. Since a higher caliber student generates stochastically higher signals, $\alpha_{i}(x)$ is increasing in $x$. In fact, it is a smoothly monotone onto function - namely, it is strictly increasing and differentiable, with $0<\alpha_{1}(x)<1$, and the limit behavior $\lim _{x \rightarrow 0} \alpha_{1}(x)=0$ and $\lim _{x \rightarrow \infty} \alpha_{1}(x)=1$.

Taking the acceptance chances as given, each student of caliber $x$ faces the portfolio optimization problem of $\S 3.1$. She must choose a set $S(x)$ of colleges to apply to, and

\footnotetext{
${ }^{6}$ For if $\alpha_{2}=1$, then $M B_{21}=c$ and $M B_{12}=c$ respectively force $\alpha_{1}=1-(c / u)$ and $\alpha_{1}=c /(1-u)$. Now, $1-(c / u)>c /(1-u)$ exactly when $c<u(1-u)$.
} 


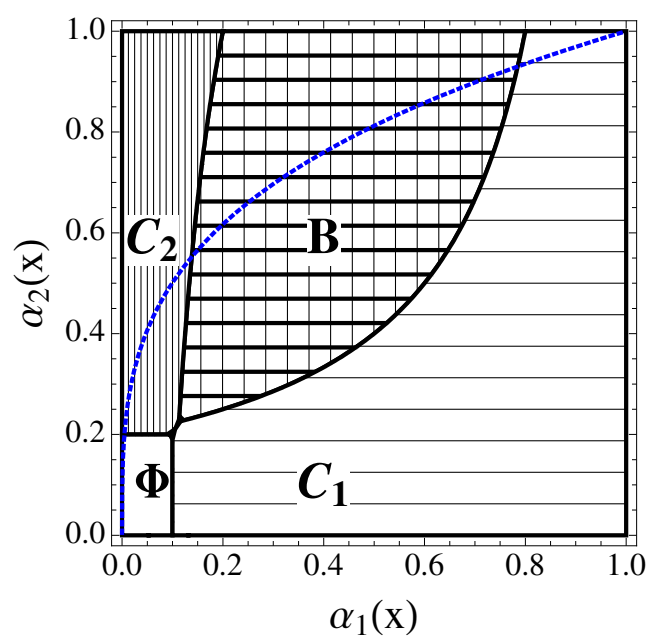

Figure 2: The Acceptance Function with Exponential Signals. The figure depicts the acceptance function $\psi\left(\alpha_{1}\right)$ for the case of exponential signals. Students apply to nowhere $(\Phi)$, college 2 only $\left(C_{2}\right)$, both colleges $(B)$ and college 1 only $\left(C_{1}\right)$ as caliber $x$ increases. Student behavior is therefore monotone for the acceptance function depicted.

accept the offer of the best school that admits her. We now translate the sets $\Phi, C_{2}, B, C_{1}$ of acceptance chance vectors into corresponding sets of calibers. Let $\mathcal{C}_{1}$ be the set of calibers that apply just to college 1 . Likewise define $\mathcal{C}_{2}$ and $\mathcal{B}$.

Key to our graphical analysis is a quantile-quantile function relating student admission chances at the colleges: Since $\alpha_{i}(x)$ strictly rises in the student's type $x$, a student's admission chance $\alpha_{2}$ to college 2 is strictly increasing in his admission chance $\alpha_{1}$ to college 1. Inverting the admission chance in the type $x$, the inverse function $\xi(\alpha, \sigma)$ is the student type accepted with chance $\alpha$ given the admission standard $\sigma$, namely $\alpha \equiv 1-G(\sigma \mid \xi(\alpha, \sigma))$. This yields an implied differentiable acceptance function

$$
\alpha_{2}=\psi\left(\alpha_{1} \mid \underline{\sigma}_{1}, \underline{\sigma}_{2}\right)=1-G\left(\sigma_{2} \mid \xi\left(\alpha_{1}, \sigma_{1}\right)\right)
$$

We prove in the appendix that the acceptance function rises in college 1's standard $\underline{\sigma}_{1}$ and falls in college 2's standard $\underline{\sigma}_{2}$, and tends to 0 and 1 as thresholds near extremes.

Observe from Figure 2 that any pair of secants drawn to a point along the acceptance function have a falling slope. To capture this, say that a function $h:[0,1] \rightarrow[0,1]$ has the double secant property if $h(\alpha)$ is a weakly increasing function on $[0,1]$ with $h(0)=0$, $h(1)=1$, and the two secant slopes $h(\alpha) / \alpha$ and $(1-h(\alpha)) /(1-\alpha)$ weakly fall in $\alpha$. This description fully captures how our acceptance chances relate to one another. 
Theorem 1 (The Acceptance Function) If $\underline{\sigma}_{1}>\underline{\sigma}_{2}$, then the acceptance function $\alpha_{2}=\psi\left(\alpha_{1}\right)$ has the double secant property. Conversely, for any smoothly monotone onto function $\alpha_{1}(x)$, and any function $h$ with the double secant property, there exists a continuous signal density $g(\sigma \mid x)$ with the strict $M L R P$, and thresholds $\underline{\sigma}_{1}>\underline{\sigma}_{2}$, for which admission chances of student $x$ to colleges 1 and 2 are $\alpha_{1}(x)$ and $h\left(\alpha_{1}(x)\right)$.

This result gives a complete characterization of how student admission chances at two ranked universities should compare. It says that if a student is so good that he is guaranteed to get into college 1 , then he is likewise a sure bet at college 2; likewise, if he is so bad that college 2 surely rejects him, then college 1 follows suit. More subtly, we arrive at the following testable implication about college acceptance chances:

Corollary 1 As a student's caliber rises, the ratio of his acceptance chances at college 1 to college 2 rises, as does the ratio of his rejection chances at college 2 to college 1.

For an example, suppose that caliber signals have the exponential density $g(\sigma \mid x)=$ $(1 / x) e^{-\sigma / x}$. The acceptance function is then given by the geometric function $\psi\left(\alpha_{1}\right)=$ $\alpha_{1}^{\underline{\sigma}_{2} / \underline{\sigma}_{1}}$, as seen in Figure 2. This is increasing and concave - and so regular - when college 2 has a lower admission standard. In turn, the acceptance relation for the location family is easily seen to be $\psi\left(\alpha_{1}\right)=1-G\left(\left(\underline{\sigma}_{2}-\underline{\sigma}_{1}\right) / \mu+G^{-1}\left(1-\alpha_{1}\right)\right)$.

\section{Equilibrium Analysis for Colleges}

\subsection{A Supply and Demand Approach}

Each college $i$ must choose an admission standard $\underline{\sigma}_{i}$ as a best response to its rival's threshold $\underline{\sigma}_{j}$ and the student portfolios. With a continuum of students, the resulting enrollment $\mathcal{E}_{i}$ at colleges $i=1,2$ is a non-stochastic number:

$$
\begin{aligned}
& \mathcal{E}_{1}\left(\underline{\sigma}_{1}, \underline{\sigma}_{2}\right)=\int_{\mathcal{B}_{\mathcal{B}} \mathcal{C}_{1}} \alpha_{1}(x) f(x) d x \\
& \mathcal{E}_{2}\left(\underline{\sigma}_{1}, \underline{\sigma}_{2}\right)=\int_{\mathcal{C}_{2}} \alpha_{2}(x) f(x) d x+\int_{\mathcal{B}} \alpha_{2}(x)\left(1-\alpha_{1}(x)\right) f(x) d x,
\end{aligned}
$$

suppressing the dependence of the sets $\mathcal{B}, \mathcal{C}_{1}$ and $\mathcal{C}_{2}$ on the student application strategy. To understand (4) and (5), observe that caliber $x$ student is admitted to college 1 with chance $\alpha_{1}(x)$, to college 2 with chance $\alpha_{2}(x)$, and finally to college 2 but not college 1 with 
chance $\alpha_{2}(x)\left(1-\alpha_{1}(x)\right)$. Also, anyone that college 1 admits will enroll automatically, while college 2 only enrolls those who either did not apply or got rejected from college 1.

If we substitute optimal student portfolios into the enrollment equations (4)-(5), then they behave like demand curves where the admissions standards are the prices. Our framework affords analogues to the substitution and income effects in demand theory. The admission rate of any student obviously falls in its anticipated admission standard the standards effect. But there is a compounding portfolio effect - that enrollment also falls due to an application portfolio shift. Each college's applicant pool shrinks in its own admissions threshold. We then deduce in the appendix the following "law of demand": If a college raises its admission standard, then its enrollment falls. Because of our portfolio effect, a college faces a more elastic demand for slots than purely predicted by the standards effect. A lower admission bar will invite applications from new students. ${ }^{7}$

The law of demand generally applies outside two college setting. For an intuition, suppose that the admissions standard at a college rises. Absent any student portfolio changes, fewer students meet its tougher admission threshold (the standards effect), and its enrollment falls. The portfolio adjustment reinforces this effect. Those who had marginally chosen to add this college to their portfolios now excise it (the portfolio effect).

In consumer demand theory, the "price" of one good affects the demand for the other, and in the two good world, they are substitutes. Analogously, we prove in the appendix, that in our model, a college's enrollment rises in its rival's admission standard. This owes to a portfolio spillover effect. If it grows tougher to gain admission to college $i$, then those who only applied to its rival continue to do so, some who were applying to both now apply just to $j$ (which helps college $j$ when it is the lesser school), and also some at the margin who applied just to $i$ now also add college $j$ to their portfolios. ${ }^{8}$

Since capacities imply vertical supply curves, we have now justified a supply and demand analysis, in which the colleges are selling differentiated products:

$$
\kappa_{1}=\mathcal{E}_{1}\left(\underline{\sigma}_{1}, \underline{\sigma}_{2}\right) \quad \text { and } \quad \kappa_{2}=\mathcal{E}_{2}\left(\underline{\sigma}_{1}, \underline{\sigma}_{2}\right)
$$

For now, we ignore the possibility that some college might not fill its capacity. Then equilibrium without excess capacity requires that both markets clear, or (6) holds. Since

\footnotetext{
${ }^{7}$ The portfolio effect may act with a lag - for instance, a college may unexpectedly ease admission standards on year, and see their applicant pool expand the next year when this becomes understood.

${ }^{8}$ As in consumer theory, complementarity may emerge with three or more goods available. With ranked colleges 1,2 , and 3 , college 3 may be harmed by tougher admissions at college 1 , if this encourages enough applications at college 2.
} 
each enrollment (demand) function is falling in its own threshold, we may invert these equations. This yields for each school $i$ the threshold that "best responds" to its rival's admissions threshold $\underline{\sigma}_{j}$ so as to fill their capacity $\kappa_{i}$ :

$$
\underline{\sigma}_{1}=\Sigma_{1}\left(\underline{\sigma}_{2}, \kappa_{1}\right) \quad \text { and } \quad \underline{\sigma}_{2}=\Sigma_{2}\left(\underline{\sigma}_{1}, \kappa_{2}\right)
$$

Given the discussion of the enrollment functions, we can treat $\Sigma_{i}$ as a "best response function" of college $i$. It rises in its rival's admission standard and falls in its own capacity. That is, the admissions standards at the two colleges are strategic complements. Figure 3 depicts an equilibrium as a crossing of these increasing best response functions.

By way of contrast, observe that without noise or without application costs, the better college is completely insulated from the actions of its lesser rival $-\Sigma_{1}$ is vertical. The equilibrium analysis is straightforward, and there is necessarily a unique equilibrium. In either case, the applicant pool of college 1 is independent of what college 2 does. For when the application signal is noiseless, just the top students apply to college 1 . And when applications are free, all students apply to college 1, and will enroll if accepted.

With application costs and noise, $\Sigma_{1}$ is upward-sloping, as application pools depend on both college thresholds. When college 2 adjusts its admission standard, the student incentives to gamble on college 1 are affected. This feedback is critical in our paper. It leads to a richer interaction among the colleges, and perhaps to multiple equilibria.

In Figure 3, the best response function $\Sigma_{1}$ is steeper than $\Sigma_{2}$ at the crossing point. Let us call any such equilibrium stable. It is robust in the following sense: Suppose that whenever enrollment falls below capacity, the college eases its admission standards, and vice versa. Then this dynamic adjustment process pushes us back towards the equilibrium. Then at this theoretical level, admission thresholds act as prices in a Walrasian tatonnement. Unstable equilibria should be rare: They require that a college's enrollment responds more to the other school's admission standard than its own.

We show in the appendix that a stable equilibrium exists, and in any such equilibrium, college 1 fills its capacity, while college 2 has excess capacity if college 1 is big enough. Surprisingly, college spaces can go unfilled despite insufficient capacity for the applicant pool. If college 1 is "too big" relative to college 2 , then college 2 is left with excess capacity. There is excess demand for college slots, and yet due to the informational frictions, there is also excess supply of slots at college 2, even at "zero price".

When college 2 has excess capacity, it optimally accepts all applicants. Since college 1 maintains an admissions standard, college behavior is monotone. But this forces $\alpha_{2}=1$ 


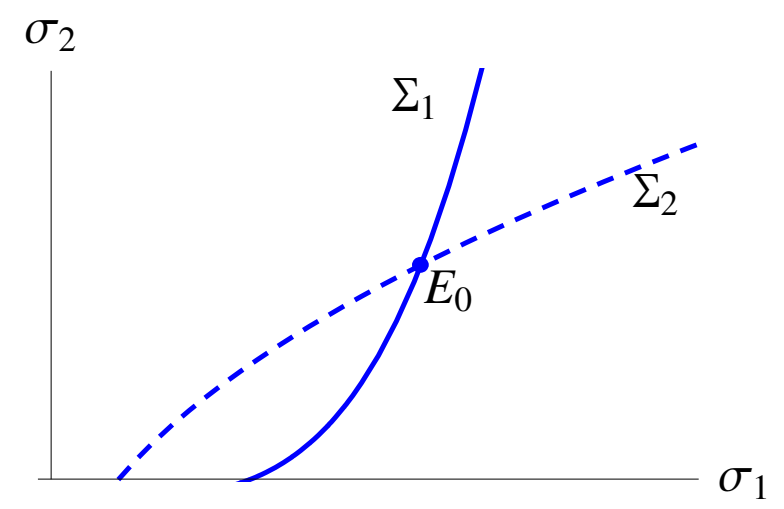

Figure 3: College Responses and a Stable Equilibrium. The functions $\Sigma_{1}$ (solid) and $\Sigma_{2}$ (dashed) give pairs of thresholds so that colleges 1 and 2 fill their capacities in equilibrium.

for all students, and so the acceptance function traverses the top side of the unit square in Figure 2. In other words, as student caliber rises, the lowest students apply to college $\{2\}$, higher students to both colleges, and the best students just apply to college $\{1\}$. Let us observe in passing that this is a sorting equilibrium.

\subsection{Changing College Sizes and Application Costs}

We now continue to explore the supply and demand metaphor, and derive some basic comparative statics. Observe that without a noisy admission process, the lesser college cannot possibly impact the better one. But with noise, the applicant pool at college 1 is sensitive to the admission threshold at college 2 , and so the best response function $\Sigma_{1}$ is positively sloped. This is the source of the novel comparative statics in our model. The lesser-ranked college imposes an "externality" upon the better college in equilibrium.

The potential multiplicity of equilibria makes a comparative statics exercise difficult. ${ }^{9}$ But fortunately, our analysis applies to all stable equilibria. Indeed, greater capacity at either college lowers both college admissions thresholds. This result speaks to the equilibrium effects at play. Greater capacity at one school, or an exogenous downward shift in the "demand" for slots, reduces the "price" (admission standard) at both schools. The graph in Figure 4 - which depicts equations (7) - constitutes the proof of part $(a)$.

For an intuition, consider a sorting equilibrium, where students apply as in Figure 2. Suppose that college 2 raises its capacity $\kappa_{2}$ (as in the right panel of Figure 4). Fixing

\footnotetext{
${ }^{9}$ It is not easy to ensure uniqueness of equilibrium. One case in which this holds is when $c$ is sufficiently small. This follows by continuity from the uniqueness of equilibrium in the costless case.
} 

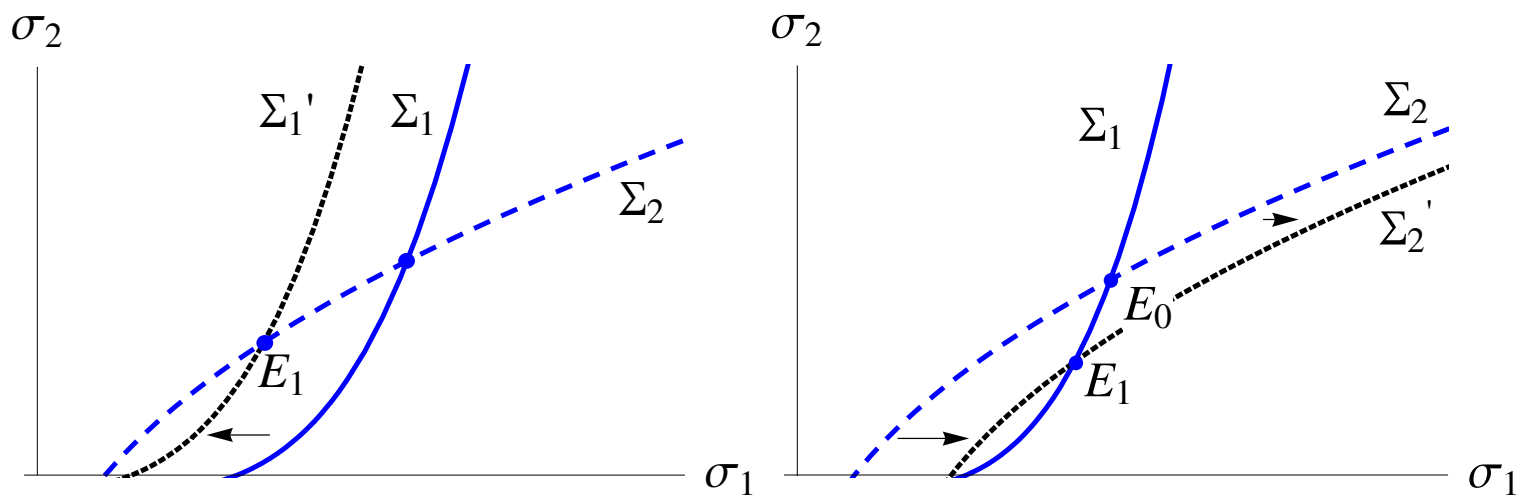

Figure 4: Equilibrium Comparative Statics. The figure illustrates how the equilibrium is affected by changing capacities $\kappa_{1}, \kappa_{2}$. The best response functions $\Sigma_{1}$ (solid) and $\Sigma_{2}$ (dashed) are drawn. The left panel considers a rise in $\kappa_{1}$, shifting $\Sigma_{1}$ left, thereby lowering both college thresholds. The right panel depicts the analogous rise in $\kappa_{2}$, and shift $\Sigma_{2}$.

the admission standard $\underline{\sigma}_{1}$, this depresses $\underline{\sigma}_{2}$. The marginal student that was indifferent between applying to college 2 only $\left(C_{2}\right)$ and both colleges $(B)$ now prefers to apply to college 2 only. So fewer apply to college 1 . Given this portfolio shift, college 1 drops its admission standards. The left panel depicts the same comparative static for college 1 .

Next, we argue that, in a sorting equilibrium, if the application cost at either college slightly falls, then the admission standard at college 1 rises and its student caliber distribution stochastically worsens. The argument is intuitive. If the application cost at college 2 falls, then more students apply, and it is forced to raise its standards. The marginal benefit (2) of a stretch application to college 1 thus rises. To counter this, college 1 responds with a higher standard, but it still gains more applicants at its lower end, and its caliber distribution stochastically worsens. By contrast, college 2 loses not only its worst students, but also top ones for whom it was insurance; its caliber change is ambiguous. Graphically, a fall in the application cost at college 2 shifts $\Sigma_{2}$ upwards without affecting $\Sigma_{1}$ - as the applicant pool at college 1 depends only indirectly on the college 2 application cost via portfolio effects as the college 2 standard changes.

The argument is more complex when the application cost at college 1 falls, since the applicant pools at both colleges depend on it. The direct impact is an expansion in the applicant pool at college 1 - consisting of those who send stretch applications to it - and a contraction in the applicant pool of college 2, triggering an increase in the admission standard at college 1 and a decrease in that of college 2 . The changes in the admissions thresholds lead to further portfolio student reallocation, and the net effect 
is a priori ambiguous. Graphically, a lower application cost at college 1 simultaneously shifts $\Sigma_{1}$ (leftwards) and $\Sigma_{2}$ (downwards). We show in the appendix that the portfolio effects at both colleges cancel out for those calibers who send stretch applications to college 1; on balance, its admission standard rises, and yet its applicant pool worsens.

The logic underlying this section does not essentially depend on the assumption that there are two colleges. For instance, whenever colleges have overlapping applicant pools, a rise in capacity at either depresses the admission standards at both.

\section{Do Colleges and Students Sort in Equilibrium?}

Casual empiricism suggests that the best students apply to and attend the best colleges, and any imperfections of the sorting owe to the noise in the application process. We now explain why both pieces of this compelling logic are false without stronger assumptions.

There are two reasons why the best students need not apply to the better college. First, as seen in Figure 5, the acceptance function may multiply cross the $M B_{12}=c$ curve, where students are indifferent between applying to both colleges and just college 2 . Such a possibility would preclude sorting: For some calibers gamble up by applying to both colleges, while higher calibers play it safe by applying to college 2 only. Figure 5 depicts a non-monotone sequence of application sets $\Phi,\{2\},\{1,2\},\{2\},\{1,2\},\{1\}$ as caliber rises. ${ }^{10}$ We show that if college 2 yields payoff $u \leq 0.5$, then this cannot happen. For then if a student applies to college 1, then any higher caliber student also does.

The next problematic case for sorting applies when college 1 is insufficiently more selective than college 2. For an extreme case, suppose that both colleges impose the same standards. In this case, the worst students who apply anywhere will choose college 1, while better students will find it worthwhile applying to both schools. This case is captured in the right panel of Figure 5. It is impossible to preclude this behavior using primitives of the student optimization alone. Rather, the college capacities must induce a sufficiently higher (endogenous) admission standard at college 1 than at college 2 .

Precluding the above two problematic cases, we show in the appendix that higher caliber students apply more aggressively if college 2 offers a sufficiently low payoff, and if it imposes a low enough admissions standard relative to college 1.

We argue in the appendix that the first condition implies that if a student applies to

\footnotetext{
${ }^{10}$ The marginal benefit $M B_{12}$ in (2) rises in the expected payoff $\alpha_{1}$ of college 1 and falls in the expected payoff $\alpha_{2} u$ of college 2. If $\alpha_{2} u$ rises faster than $\alpha_{1}$, then a better student may drop.
} 

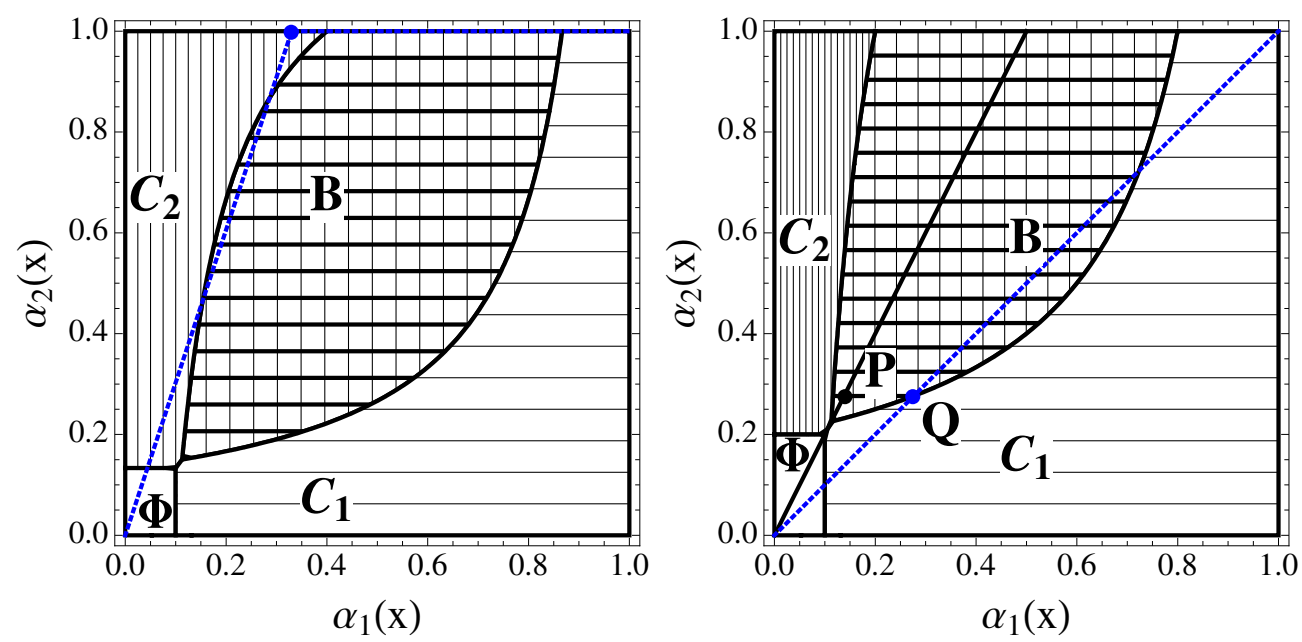

Figure 5: Non-Monotone Behavior. In the left panel, the signal structure induces a piecewise linear acceptance function. Student behavior is non-monotone, since there are both low and high caliber students who apply to college 2 only $\left(C_{2}\right)$, while intermediate ones insure by applying to both. In the right panel, equal thresholds at both colleges induce an acceptance function along the diagonal, $\alpha_{1}=\alpha_{2}$. Student behavior is non-monotone, as both low and high caliber students apply to college 1 only $\left(C_{1}\right)$, while middling caliber students apply to both. Such an acceptance function also arises when caliber signals are very noisy.

college 1 , then so does any better student. Intuitively, the marginal benefit of applying to college 1 in addition to college 2 falls in the quality of this lesser foregone alternative. Further, Theorem 1 argues that a better student has a proportionately better chance of gaining admission to college 1. The appendix combines these two insights and deduces that when college 2 has a low enough payoff $u$, a better student always has a greater marginal gain from adding college 1. Summarizing, this case addresses "stretch applications", ensuring that the acceptance function traverses region $C_{2}$ before region $B$.

The next condition instead turns on "safety schools" - graphically, that the acceptance function hits region $B$ before region $C_{1}$. We show that if college 2 imposes a low enough admission standard, then the weakest students will always first choose it. And as noted, better students have proportionately better admission chances at college 1 than college 2 (Theorem 1). From these two insights we deduce in the appendix that when any student portfolio includes college 2 , then no worse student applies just to college 1 .

So inspired, we next provide sufficient conditions on the fundamentals - college capacity and quality - that deliver sorting in equilibrium for colleges and students. We show that these conditions are needed if sorting emerges for any signal distribution. 


\section{Theorem 2 (Non-Sorting and Sorting in Equilibrium)}

(a) If college 2 is "too good" (i.e., $u>0.5$ ), then there exists a continuous MLRP density $g(\sigma \mid x)$ that yields a stable equilibrium with non-monotone student behavior.

(b) If college 2 is not too big relative to college 1 , then college 2 sets a higher admissions standard than college 1 in some equilibrium.

(c) If college 1 is not too big relative to college 2 , and college 2 is not too good (namely, $u \leq 0.5)$, then there are only sorting equilibria and neither college has excess capacity.

We tackle part $(a)$ constructively in the appendix, starting with the acceptance function depicted in the left panel of Figure 5 and then appealing to Theorem 1 to find some signal distribution that generates it. The message of part $(b)$ is that even $a$ bad college 2 may maintain higher standards if it is sufficiently small. In other words, admissions standards can be misleading measures of college quality — and this should cast doubts on college rankings computed based on this measure. Because college 2 has higher admissions standards and yields a lower payoff, it is no applicant's first choice. But some students may yet insure themselves with an application to college 2 . If it is sufficiently small, then it may entirely fill its capacity with these insurance applicants.

All told, parts $(a)$ and $(b)$ provide conditions under which sorting fails in some equilibrium. Part $(c)$ concludes with sufficient conditions for sorting in all equilibria.

\section{The Spillover Effects of Affirmative Action}

We now slightly enrich our model, and address the topical issue of affirmative action in college admissions. Specifically, we explore how affirmative action at one college has spillover effects at the other. We first assume that a fraction $\phi$ of the applicant pool belongs to a target group. This may well be a under-represented minority, but it may also be a majority group. For instance, many states favor their own students at state colleges - Wisconsin public colleges can have at most $25 \%$ out-of-state students. Just as well, some colleges strongly value athletes. We assume a common caliber distribution, so that there is no other reason for differential treatment of the applicants.

Assume that students honestly report their "target group" status on their applications. Moreover, assume that students from both groups use monotone application strategies. Reflecting the colleges' desire for a more diverse student body, let college $i$ earn a bonus $\pi_{i} \geq 0$ for each enrolled target student. The colleges may set different 
thresholds for the two groups. Let colleges 1,2 offer a "discount" $\Delta_{1}, \Delta_{2}$ to target applicants. In other words, the respective standards for non-target and target groups are $\left(\underline{\sigma}_{1}, \underline{\sigma}_{2}\right)$ and $\left(\underline{\sigma}_{1}-\Delta_{1}, \underline{\sigma}_{2}-\Delta_{2}\right)$. At each college, the expected payoff of the marginal admits from the two groups should coincide - except of course at a corner solutions (when a college admits all students from a group). This yields two new equilibrium conditions that account for the fact that ex post, colleges behave rationally, and equate their expected values of target and non-target applicants.

$$
\begin{aligned}
E\left[X+\pi_{1} \mid \sigma=\underline{\sigma}_{1}-\Delta_{1}, \text { target }\right] & =E\left[X \mid \underline{\sigma}_{1}, \text { non-target }\right] \\
E\left[X+\pi_{2} \mid \sigma=\underline{\sigma}_{2}-\Delta_{2}, \text { target, accepts }\right] & =E\left[X \mid \underline{\sigma}_{2}, \text { non-target, accepts }\right]
\end{aligned}
$$

Here, $X$ is the student caliber. So as with third degree price discrimination, colleges equate the shadow cost of capacity across groups. Along with market clearing (6) at each college, equilibrium requires solving four equations in four unknowns.

For any discounts $\Delta_{1}, \Delta_{2}$, we let $\left(\underline{\sigma}_{1}\left(\Delta_{1}, \Delta_{2}\right), \underline{\sigma}_{2}\left(\Delta_{1}, \Delta_{2}\right)\right)$ be admission standards for non-target students that fill the capacity at both colleges - i.e. solving the equations (6). As in Section 4, there could be multiple solutions. Consider a stable one. ${ }^{11}$ Then let $V_{i}\left(\Delta_{1}, \Delta_{2}, \pi_{i}\right)$ be the shadow value difference in the LHS and RHS of (8)-(9), evaluated at capacity-filling standards $\left(\underline{\sigma}_{1}, \underline{\sigma}_{2}\right)$. An equilibrium is then a zero $V_{1}=V_{2}=0$. Naturally, without any group preference $\left(\pi_{1}=\pi_{2}=0\right)$, an equilibrium is $\Delta_{1}=\Delta_{2}=0$. Let us now define two new college best response functions. Write $\Delta_{i}=\Upsilon_{i}\left(\Delta_{j}, \pi_{i}\right)$ when $V_{i}\left(\Delta_{1}, \Delta_{2}, \pi_{i}\right)=0$. An equilibrium is then a crossing point of $\Upsilon_{1}, \Upsilon_{2}$ in $\left(\Delta_{1}, \Delta_{2}\right)$-space.

It is a priori not clear what happens to the equilibrium as the group preferences $\pi_{1}$ and $\pi_{2}$ change. This hinges on the sign of the derivatives of $V_{i}$ with respect to $\Delta_{1}$ and $\Delta_{2}$. To see the difficulty, consider for example what happens to $V_{1}$ after the discount $\Delta_{1}$ at college 1 rises. The immediate effect is that $V_{1}$ falls, as target students meet a lower standard - fixing the non-target standards. But there are two feedback due to capacity considerations alone. When the discount $\Delta_{i}$ for a target student at college $i$ changes, there is an indirect effect — operating through the capacity equations - on the non-target standard at college $j$. In the appendix, we show that this subtle feedback are negligible locally around $\Delta_{1}=\Delta_{2}=0$ with no affirmative action. From now on, we ignore these two cross feedback effects in computing the total derivatives in $\Delta_{1}, \Delta_{2}$.

It is critical to pin down the slopes of $\Upsilon_{1}$ and $\Upsilon_{2}$. From college 1 's perspective, shadow

\footnotetext{
${ }^{11} \mathrm{~A}$ simple modification of the proof of Claim 2 appendix gives existence of such a stable solution.
} 
value equalization requires that the discounts $\Delta_{1}$ and $\Delta_{2}$ rise or fall together. Why? We argue that these discounts have opposite effects on the shadow value difference $V_{1}$.

Lower standards for target students at college 1 not only depresses their average caliber via the standards effect, but also encourages worse target applicants to apply i.e. the portfolio effect reinforces this. To fill capacity, the non-target student standard must rise at college 1; their quality rises due to the portfolio and standards effects. Altogether, the shadow value of non-target students rises relative to target students. Conversely, lower standards for target students at college 2 deters the weakest target "stretch" applicants at college 1, via the portfolio effect. So ignoring the cross effects, the shadow value difference $V_{1}$ rises in $\Delta_{2}$. To summarize, to maintain (8), an increase in $\Delta_{1}$ must be accompanied by an increase in the discount $\Delta_{2}$, and thus $\Upsilon_{1}$ slopes up.

By contrast, the slope of the $\Upsilon_{2}$ is ambiguous. First, when college 1 favors some students more, the portfolio and standards effects reinforce. College 2 loses some stellar target "safety" applicants, but the remaining top tier of target applicants gain admission to college 1 more often, and so are unavailable to college 2. Moreover, the pool of nontarget applicants at college 2 improves since their admission standard rises to meet the capacity constraint. In short, the shadow value difference of target students less that of non-target students falls. But this difference may rise or fall when college 2 favors target students more. The standards effect is negative, but the portfolio effect is ambiguous: Its favored applicant pool expands at the lower and upper ends.

We now resolve this indeterminacy and argue that the slope of the $\Upsilon_{2}$ is negative at the stable equilibria. Assume that when the shadow value of a target student exceeds that of a non-target student, college $i$ responds by raising the target advantage $\Delta_{i}$. Call the equilibrium shadow value stable if this dynamic adjustment process pushes us back to the equilibrium. We argue that for any such equilibrium, the shadow value difference of targeted over non-targeted students falls when college 2 favors the targeted students more. For suppose not. Then we must have $V_{2}>0$ whenever $\left(\Delta_{1}, \Delta_{2}\right)$ lies above the $\Upsilon_{2}$ schedule (along which $V_{2}=0$ ). Thus, the adjustment process would lead to an even higher $\Delta_{2}$, contrary to shadow value stability. Figure 6 illustrates this logic. So $V_{2}$ must fall in $\Delta_{2}$ at a shadow value stable equilibrium; therefore, $\Upsilon_{2}$ slopes down near any such equilibrium, as in the right panel of Figure 6.

We are now ready to state the following result, whose proof is depicted graphically. 

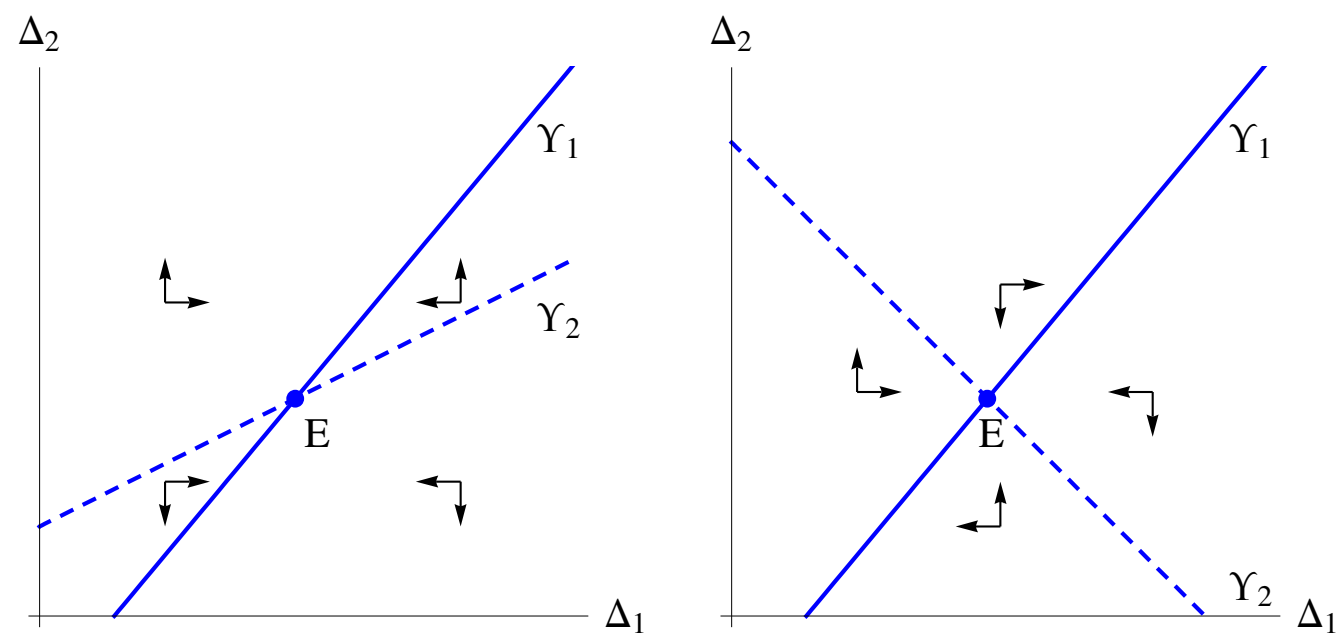

Figure 6: Shadow Value Stable Equilibrium. The left panel illustrates a shadow value unstable equilibrium, which happens when the best response $\Upsilon_{2}$ slopes upward. A necessary condition for shadow value stability is that $\partial V_{2} / \partial \Delta_{2}$ be negative, thus ensuring that $\Upsilon_{2}$ slopes downward. The right panel depicts a shadow value stable equilibrium.

Theorem 3 (Affirmative Action) Fix $\pi_{1}=\pi_{2}=0$. Assume that $\Delta_{1}=\Delta_{2}=0$ is a shadow value stable equilibrium with monotone student behavior ${ }^{12}$ As the preference for a target group at college 1 rises, it favors those students and college 2 penalizes them. As the preference for target students at college 2 rises, both colleges favor them more.

Let's highlight here an asymmetry. Affirmative action for target students by college 2 leads college 1 to follow suit. But affirmative action for target students at college 1 induces college 2 to penalize them. Two effects underpin this asymmetry. First, consider the acceptance curse facing college 2. A student who enrolls at college 2 either just applied there, or was strong enough to have applied to college 1 also - and was rejected. So the event that a student enrolls at college 2 is a worse signal if college 1 has favored them. College 2 must counters this with a penalty, which is further aggravated by a portfolio effect: Indeed, the best favored students that previously applied to college 2 now just apply to college 1; therefore, the pool of favored applicants at college 2 worsens.

\section{$7 \quad$ Early Admissions}

In a final illustration of how student portfolio choice makes colleges interdependent, we consider early admissions for students. We find a close parallel with the analysis of

\footnotetext{
${ }^{12}$ Such an equilibrium easily exists when $c=0$, and by continuity for $c$ small enough.
} 

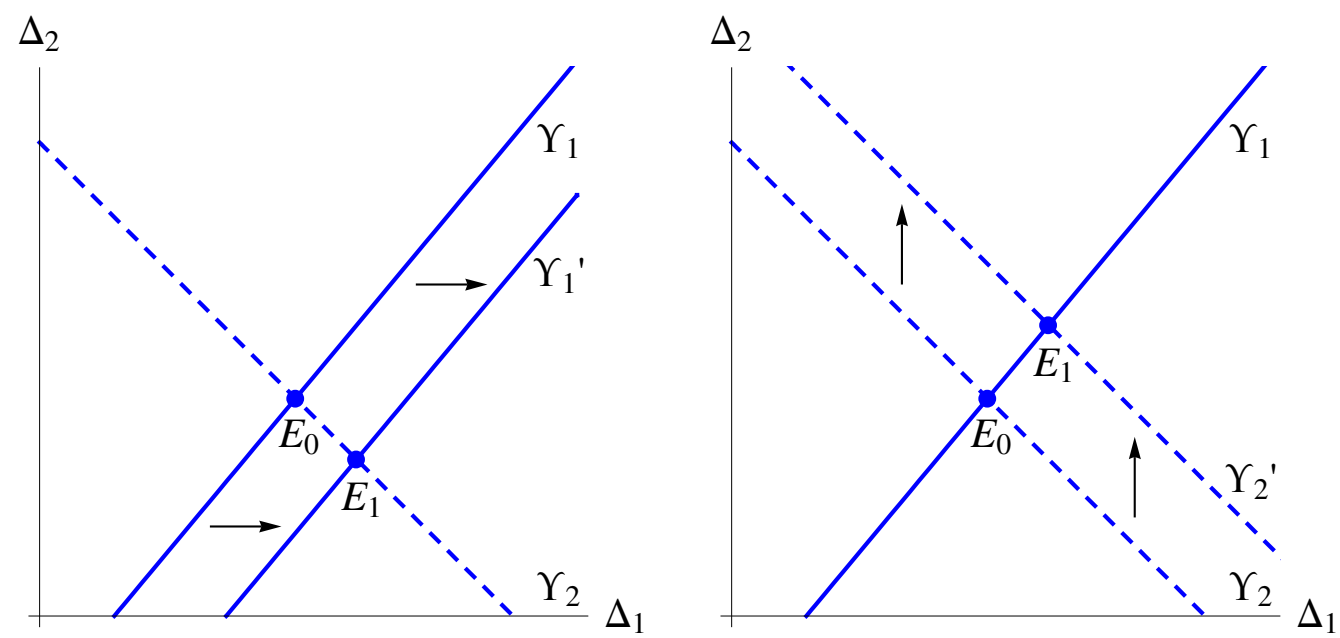

Figure 7: Affirmative Action Comparative Statics. The left panel depicts a right shift of the best response discount curve $\Upsilon_{1}$ as the minority preference $\pi_{1}$ increases. The equilibrium shifts to $E_{1}$, with a higher discount $\Delta_{1}$ and a lower $\Delta_{2}$. This is justified for small minority preferences in Theorem 3 . The right panel depicts how the best response $\Upsilon_{2}$ shifts up in $\pi_{2}$, increasing both equilibrium discounts $\Delta_{1}$ and $\Delta_{2}$.

affirmative action. We assume that in addition to applying in the regular period, students can also apply early. To stay focused, we analyze "early action" rather than early decision, namely, non-binding early admission. We also assume that only one college has an early admissions policy, and that its rejection is final: Applicants are not "deferred". While we can shed light on this important topic, we observe that this restriction precludes the very difficult analysis of competing early admissions programs between colleges. As in the analysis of affirmative action, college $i$ may wish to discriminate between early and regular applicants. So specifically, we assume an early standard $\underline{\sigma}_{i}-\Delta_{i}$ and a regular standard $\underline{\sigma}_{i}$, where the early "discount" $\Delta_{i}$ may well be negative.

For our first insight, we consider the student application problem. Loosely, other things equal, applying early is better. Formally, if college $i$ offers early admissions, and does not penalize early applicants (so that $\Delta_{i} \geq 0$ ), then an early application to it is weakly preferable to a regular application. Indeed, this is clear if just one regular application is planned. Any student planning two regular applications can do better by applying first to college $i$ and then to college $j$ in the regular period (if he so desires).

The equilibrium analysis requires an additional optimality condition upon the college offering early admission. If it admits students in both early and regular periods, then the expected caliber of the marginal enrollee in the two periods must coincide. In this 
way, we draw inspiration from the affirmative action section. By the logic of (8) and (9):

$$
E\left[X \mid \sigma=\underline{\sigma}_{i}-\Delta_{i}, \text { applies early, accepts }\right]=E\left[X \mid \sigma=\underline{\sigma}_{i}, \text { applies regular, accepts }\right]
$$

Namely, the shadow values of early and late applicants are equalized. To proceed, we first suppose that a representative fraction $\mu \in(0,1)$ of applicants has a "coupon" that allows them to apply early, if they wish; thus, there always remain regular applicants, and so (10) holds. Since $\mu$ can be very near 1 , this assumption is weak. We assume monotone student behavior in our standard setting without an early admission option.

\section{Theorem 4 (Early Admissions) Assume monotone student behavior.}

(a) If college 1 has an early admissions program, then it penalizes early applicants.

(b) In some equilibrium, college 2 favors early applicants, poaching from college 1.

The argument for part $(a)$ is instructive. Suppose instead that it weakly favors them. By our previous logic about early admissions at college 1, any students that would apply in the regular period will apply early, but also, some lower caliber students are induced to apply early. This is necessarily a lower set of students, with a lower shadow value, than in the regular period - namely, those students without coupons. This violates the required optimality condition (10). So college 1 must compensate by penalizing early applicants. Intuitively, if the early standards are weakly lower, then portfolio and standards effects reinforce each other, depressing the shadow value of early applicants.

We next turn to the more interesting case in part (b) when college 2 offers the early admission program. We show that this enables it to "poach" students from college 1 namely, some students who would have applied to both colleges regular, will only apply to college 2 early if they have a coupon, and will forego an extra application to college 1. To see this, suppose that college 2 favors early applicants, with a discount $\Delta_{2} \geq 0$. Since any student then enjoys a weakly greater admission chance early than regular, he will apply early by our previous discussion. Also, of the students admitted at college 2, some will then drop their regular application to college 1. Recalling the marginal benefit (2), this incentive exists for students whose regular admission chances $\alpha_{1}^{R}, \alpha_{2}^{R}$ satisfy

$$
\alpha_{1}^{R}(x)(1-u)<c<\alpha_{1}^{R}\left(1-\alpha_{2}^{R} u\right)
$$

This inequality is possible because the marginal gain of applying regular to college 1 is smaller if one has already gained admission at college 2. This suggested behavior - 

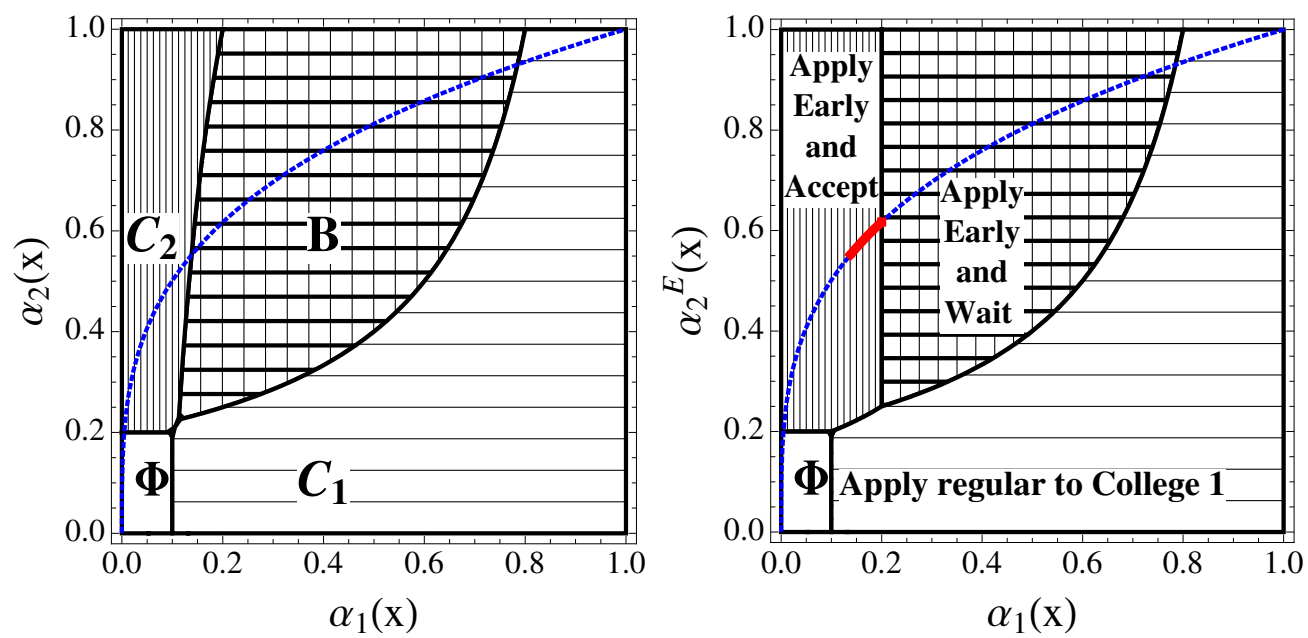

Figure 8: Capturing Students with Early Action. The left and right panels depict the optimal student strategy regions under regular and early action programs, respectively. The key difference is the set of types on the solid (red) part of the acceptance function in the right panel: Students without coupons apply to both colleges and accept college 1 if accepted, but those with coupons apply early to college 2 and don't bother to apply to college 1 if accepted. College 2 successfully pre-empts college 1 , and poaches the students.

depicted in Figure 8 - obtains in some equilibrium.

These insights are also robust beyond the two college world, where just one college offers early admissions. If that college is the best, then it should penalize applicants. If it is a lesser school, then it can profitably poach students from better colleges.

\section{Concluding Remarks}

We have reframed the college admissions problem with frictions: applications are costly, students heterogeneous, and evaluations noisy. We have related student admission chances at two ranked colleges, and built a graphical apparatus for analyzing student and college choice. The student optimization introduces the distinction between stretch and safety schools. In this Bayesian setting, admissions standards act as prices. College enrollment obeys the law of demand not only because fewer students meet a tougher admission standard, but also since not as many apply when the admission prospects are not as bright. This portfolio effect raises the demand elasticity for student slots at a college beyond that explained by the standards. This means, e.g., that a college might meet a major enrollment mandate for target students with a minor standards shift.

We have shown that frictions may preclude sorting of students and colleges through 
two channels. First, better students need not apply more aggressively: For if the worse college is either too good or too small, or the application process is noisy enough, one student may gamble on the better college while a more talented one does not. Second, college admissions standards needn't reflect their quality - the worse college may set higher standards if it is small enough. Large public schools might well be punished in college rankings publications that use SAT scores of enrolled students in ranking schools.

We have also found how the applications frictions induce interdependencies among the colleges: That lesser colleges can impact better rivals is an important and realistic novelty missing from frictionless models of student-college matching. For instance, when its lesser rival raises its capacity or lowers its application cost, the better one should lower its admission standard and will see its student body composition shift.

One strength of our model is that it affords a unified treatment of a wide array of seemingly unrelated questions about college admissions. Indeed, by allowing differentiated consumer groups, we have also explored affirmative action and early admissions. This richer model reveals that private colleges might wisely discriminate against state residents. And we would argue that some top colleges have dropped early admissions programs since it encourages aggressive applications by undesirable marginal students.

Tractability has forced us to assume common student preferences and restrict to two colleges. Relaxing these restrictions is an important open problem. Our model is also amenable to structural estimation, and $\mathrm{Fu}$ (2009) has taken a first step in this direction.

\section{A Appendix: Proofs}

\section{A.1 Colleges Optimally Employ Admissions Thresholds}

Let $\chi_{i}(\sigma)$ be the expected value of the student's caliber given that he applies to college $i$, his signal is $\sigma$, and he accepts. College $i$ optimally employs a threshold rule if, and only if, $\chi_{i}(\sigma)$ increases in $\sigma$. For college 1 this is immediate, since $g(\sigma \mid x)$ enjoys the MLRP property. We prove this for college 2 , since it faces an acceptance curse. We assume that students of calibers in set $\mathcal{C}_{i}$ apply to college $i$ only, and in $\mathcal{B}$ apply to both colleges. ${ }^{13}$

$$
\chi_{2}(\sigma)=\frac{\int_{\mathcal{C}_{2}} x g(\sigma \mid x) f(x) d x+\int_{\mathcal{B}} x G\left(\underline{\sigma}_{1} \mid x\right) g(\sigma \mid x) f(x) d x}{\int_{\mathcal{C}_{2}} g(\sigma \mid x) f(x) d x+\int_{\mathcal{B}} G\left(\underline{\sigma}_{1} \mid x\right) g(\sigma \mid x) f(x) d x}
$$

\footnotetext{
${ }^{13}$ We assume that students employ pure strategies, which follows from our analysis of the student optimization in $\S 3.1$. Measurability of sets $\mathcal{B}$ and $\mathcal{C}_{2}$ owe to the continuity of our functions $\alpha_{i}(x)$ in $\S 3.2$.
} 
It is easy to show that $\chi_{2}(\sigma)$ is less that the expectation without the cdf's $G$ - because being accepted by a student reduces college 2's estimate of his caliber, as there is a positive probability that the student was rejected by college 1; i.e., college 2 suffers an acceptance curse effect. Write $(12)$ as $\chi_{2}(\sigma)=\int_{\mathcal{B}_{\mathcal{L C}}} x h_{2}(x \mid \sigma) d x$ using indicator function notation:

$$
h_{2}(x \mid \sigma)=\frac{\left(I_{\mathcal{C}_{2}}(x)+I_{\mathcal{B}} G\left(\underline{\sigma}_{1} \mid x\right)\right) g(\sigma \mid x) f(x)}{\int_{\mathcal{B} \cup \mathcal{C}_{2}}\left(I_{\mathcal{C}_{2}}(t)+I_{\mathcal{B}} G\left(\underline{\sigma}_{1} \mid t\right)\right) g(\sigma \mid t) f(t) d t},
$$

Then the 'density' $h_{2}(x \mid \sigma)$ has the MLRP. Therefore, $\chi_{2}(\sigma)$ increases in $\sigma$.

\section{A.2 Simultaneous versus Sequential Timing}

We claim that the subgame perfect equilibrium (SPE) outcomes of the two-stage game with students moving first coincide with the Nash equilibria of the one-shot game.

First, consider the outcome of a SPE of the two-stage game, where students choose application $S=S(\cdot)$ and then colleges choose standards $\underline{\sigma}_{1}(S)$ and $\underline{\sigma}_{2}(S)$. Then colleges must be best responding to each other and to $S$ (since $S$ is realized when they choose). Also, students can forecast how colleges would respond to $S$ in an SPE of the two-stage game, and so their applications must be best replies to the standards $\underline{\sigma}_{1}(S)$ and $\underline{\sigma}_{2}(S)$. Thus, it is an equilibrium of the one-shot game.

Conversely, since each student has measure zero, he cannot affect the college standards by adjusting his application strategy. Hence, any equilibrium $\left(S, \underline{\sigma}_{1}(S), \underline{\sigma}_{2}(S)\right)$ of the one-shot game is also the outcome an SPE of the two-stage game.

\section{A.3 Acceptance Function and Signals}

Since $G\left(\underline{\sigma}_{1} \mid x\right)$ is continuously differentiable in $x$, the acceptance function is continuously differentiable on $(0,1]$. Given $\alpha \equiv 1-G(\sigma \mid \xi(\alpha, \sigma))$, partial derivatives have positive slopes $\xi_{\alpha}, \xi_{\sigma}>0$. Differentiating (3),

$$
\begin{aligned}
\frac{\partial \psi}{\partial \alpha_{1}} & =-G_{x}\left(\underline{\sigma}_{2} \mid \xi\left(\alpha_{1}, \underline{\sigma}_{1}\right)\right) \xi_{\alpha}\left(\alpha_{1}, \underline{\sigma}_{1}\right)>0 \\
\frac{\partial \psi}{\partial \underline{\sigma}_{1}} & =-G_{x}\left(\underline{\sigma}_{2} \mid \xi\left(\alpha_{1}, \underline{\sigma}_{1}\right)\right) \xi_{\sigma}\left(\alpha_{1}, \underline{\sigma}_{1}\right)>0 \\
\frac{\partial \psi}{\partial \underline{\sigma}_{2}} & =-g\left(\underline{\sigma}_{2} \mid \xi\left(\alpha_{1}, \underline{\sigma}_{1}\right)\right)<0
\end{aligned}
$$


Properties of the cdf $G$ imply $\psi\left(0, \underline{\sigma}_{1}, \underline{\sigma}_{2}\right) \geq 0$ and $\psi\left(1, \underline{\sigma}_{1}, \underline{\sigma}_{2}\right)=1$. The limits of $\psi$ as thresholds approach the supremum and infimum owe to limit properties of $G$.

\section{A.4 Acceptance Function Shape: Proof of Theorem 1}

$(\Rightarrow)$ The Acceptance Function is Regular. First, $G(\sigma \mid x)$ and $1-G(\sigma \mid x)$ are strictly log-supermodular in $(\sigma, x)$ since the density $g(\sigma \mid x)$ obeys the strict MLRP. Since $x=\xi\left(\alpha_{1}, \underline{\sigma}_{1}\right)$ is strictly increasing, $G\left(s \mid \xi\left(\alpha_{1}, \underline{\sigma}_{1}\right)\right)$ and $1-G\left(s \mid \xi\left(\alpha_{1}, \underline{\sigma}_{1}\right)\right)$ are then strictly $\log$-supermodular in $\left(s, \alpha_{1}\right)$. So the secant slopes below strictly fall in $\alpha_{1}$, since $\underline{\sigma}_{1}>\underline{\sigma}_{2}$ :

$$
\frac{\psi\left(\alpha_{1}\right)}{\alpha_{1}}=\frac{1-G\left(\underline{\sigma}_{2} \mid \xi\left(\alpha_{1}\right)\right)}{1-G\left(\underline{\sigma}_{1} \mid \xi\left(\alpha_{1}\right)\right)} \quad \text { and } \quad \frac{1-\psi\left(\alpha_{1}\right)}{1-\alpha_{1}}=\frac{G\left(\underline{\sigma}_{2} \mid \xi\left(\alpha_{1}\right)\right)}{G\left(\underline{\sigma}_{1} \mid \xi\left(\alpha_{1}\right)\right)}
$$

$(\Leftarrow)$ Deriving a Signal Distribution. Conversely, fix a regular function $h$ and a smoothly monotone onto function $\alpha_{1}(x)$. Also, put $\alpha_{2}(x)=h\left(\alpha_{1}(x)\right)$, so that $\alpha_{2}(x)>\alpha_{1}(x)$. We must find a continuous signal density $g(\sigma \mid x)$ with the strict MLRP and thresholds $\sigma_{1}>\sigma_{2}$ that rationalizes the $h$ as the acceptance function consistent with these thresholds and signal distribution.

Step 1: A Discrete Signal Distribution. Consider a discrete distribution with realizations in $\{-1,0,1\}: g_{1}(x)=\alpha_{1}(x), g_{0}(x)=\alpha_{2}(x)-\alpha_{1}(x)$ and $g_{-1}(x)=1-\alpha_{2}(x)$. Indeed, for each caliber $x, g_{i} \geq 0$ and sum to 1 . This obeys the strict MLRP because

$$
\frac{g_{0}(x)}{g_{1}(x)}=\frac{\alpha_{2}(x)-\alpha_{1}(x)}{\alpha_{1}(x)}=\frac{h\left(\alpha_{1}(x)\right)}{\alpha_{1}(x)}-1
$$

is strictly decreasing by the first secant property of $h$, and

$$
\frac{g_{0}(x)}{g_{-1}(x)}=\frac{\alpha_{2}(x)-\alpha_{1}(x)}{1-\alpha_{2}(x)}=-1+\frac{1-\alpha_{1}(x)}{1-h\left(\alpha_{1}(x)\right)}
$$

is strictly increasing in $x$ by the second secant property of $h$.

Let the college thresholds be $\left(\underline{\sigma}_{1}, \underline{\sigma}_{2}\right)=(0.5,-0.5)$. Then $G\left(\underline{\sigma}_{1} \mid x\right)=g_{-1}(x)+$ $g_{0}(x)=1-\alpha_{1}(x)$ and $G\left(\underline{\sigma}_{2} \mid x\right)=g_{-1}(x)=1-\alpha_{2}(x)$. Rearranging yields $\alpha_{1}(x)=$ $1-G\left(\underline{\sigma}_{1} \mid x\right)$ and $\alpha_{2}(x)=1-G\left(\underline{\sigma}_{2} \mid x\right)$. Inverting $\alpha_{1}(x)$ and recalling that $\alpha_{2}=h\left(\alpha_{1}\right)$, we obtain $\alpha_{2}=h\left(\alpha_{1}\right)=1-G\left(\underline{\sigma}_{2} \mid \xi\left(\underline{\sigma}_{1}, \alpha_{1}\right)\right)$, thereby showing that $h$ is the acceptance function consistent with this signal distribution and thresholds.

Step 2: A Continuous Signal Density. To create an atomless signal distribution, we smooth this example using the triangular kernel $k(s)=\max \{1-s, 0\}$. 
Fix $\beta>0$, and define $g(\sigma \mid x)=\beta \sum_{i=\{-1,0,1\}} g_{i}(x) k(\beta(\sigma-i))$. For any "bandwidth" $1 / \beta \in(0,1 / 2)$, acceptance chances remain the same as with the discrete signals, since the masses at $\{-1,0,1\}$ is not transferred past the (respective) thresholds $\{0.5,-0.5\}$.

The strict MLRP implies that $g_{i}(x)$ is strictly log-supermodular in $(i, x)$. Also, the function $k(s)$ is concave in $s$, and thus log-concave in $s$ too. This implies that $k(\beta(\sigma-i))$ is log-supermodular in $(i, \sigma)$. This is in $\S 1.5$ in Karlin (1968), but for a self-contained treatment, let's assume twice differentiability: Then $k_{\sigma i} k-k_{\sigma} k_{i}>0$ iff $-k^{\prime \prime} k+k^{\prime} k^{\prime}>0$, which holds iff $k$ is $\log$ concave. Thus, $g_{i}(x) k(\beta(\sigma-i))$ is log-supermodular in $(i, x, \sigma)$. Finally, partially summing over $i=1,2,3$ yields a log-supermodular function of $(x, \sigma)$, by Proposition 3.2 in Karlin and Rinott (1980) - it is the MRLP property.

\section{A.5 Monotone Student Strategies}

Lemma 1 (Monotone Applications) Student behavior is monotone in caliber if

(a) College 2 has payoff $u \leq 0.5$, so that if a student applies to college 1 , then any better student will also apply to college 1 , and

(b) College 2 imposes a low enough admissions standard relative to college 1 so that if a student applies to college 2, then any worse student applies to college 2 or nowhere.

The proof proceeds as follows. First, we show that $u \leq 0.5$ implies that if a caliber applies to college 1, any higher caliber applies as well. Second, we produce a sufficient condition that ensures that the admissions threshold at college 2 is sufficiently lower than that of college 1 , so that if a caliber applies to college 2, then any lower caliber who applies to college sends an application to college 2, and calibers at the lower tail apply nowhere. From these two results, monotone student behavior ensues.

Proof of PART $(a)$, Step 1. We first show that the acceptance function $\alpha_{2}=$ $\psi\left(\alpha_{1}\right)$ crosses $\alpha_{2}=1 / u\left(1-c / \alpha_{1}\right)$ (i.e., $M B_{12} \equiv \alpha_{1}\left(1-\alpha_{2} u\right)=c$ ) only once when $u \leq 0.5$. Since $(i)$ the acceptance function starts at $\alpha_{1}=0$ and ends at $\alpha_{1}=1$, (ii) $M B_{12}=c$ starts at $\alpha_{1}=c$ and ends at $\alpha_{1}=c /(1-u)$, and $(i i i)$ both functions are continuous, there exists a crossing point. Clearly, they intersect when $\alpha_{1}\left(1-\psi\left(\alpha_{1}\right) u\right)=c$. Now,

$\left[\left(1-\psi\left(\alpha_{1}\right) u\right) \alpha_{1}\right]^{\prime}=1-u \psi\left(\alpha_{1}\right)-\alpha_{1} u \psi^{\prime}\left(\alpha_{1}\right)>1-u \psi\left(\alpha_{1}\right)-u \psi\left(\alpha_{1}\right)=1-2 u \psi\left(\alpha_{1}\right) \geq 1-2 u \geq 0$,

where the first inequality exploits $\psi\left(\alpha_{1}\right) / \alpha_{1}$ falling in $\alpha_{1}$ (Theorem 1), i.e. $\psi^{\prime}\left(\alpha_{1}\right)<$ $\psi\left(\alpha_{1}\right) / \alpha_{1}$; the next two inequalities use $\psi\left(\alpha_{1}\right) \leq 1$ and $u \leq 0.5$. Since $M B_{12}$ is rising in $\alpha_{1}$ when the acceptance relation hits $\alpha_{2}=\left(1-c / \alpha_{1}\right) / u$, the intersection is unique. 
Proof of Part (a), Step 2. We now show that Step 1 implies the following single crossing property in terms of $x$ : if caliber $x$ applies to college 1 (i.e., if $1 \in S(x)$, then any caliber $y>x$ also applies to college 1 (i.e., $1 \in S(y)$ ). Suppose not; i.e., assume that either $S(y)=\varnothing$ or $S(y)=\{2\}$. If $S(y)=\varnothing$, then $S(x)=\varnothing$ as well, as $\alpha_{1}(x)<\alpha_{1}(y)$ and $\alpha_{2}(x)<\alpha_{2}(y)$, contradicting the hypothesis that $1 \in S(x)$. If $S(y)=\{2\}$, then there are two cases: $S(x)=\{1\}$ or $S(x)=\{1,2\}$. The first cannot occur, for by Theorem $1 \alpha_{2}(x) / \alpha_{1}(x)>\alpha_{2}(y) / \alpha_{1}(y)$, and thus $\alpha_{2}(y) u \geq \alpha_{1}(y)$ implies $\alpha_{2}(x) u>\alpha_{1}(x)$, contradicting $S(x)=\{1\}$. In turn, the second case is ruled out by the monotonicity of $M B_{12}$ derived above, as caliber $y$ has greater incentives than $x$ to add college 1 to its portfolio, and thus $S(y)=\{2\}$ cannot be optimal.

Proof of PART (b), Step 1. We first show that if the acceptance function passes above the point $\left(\bar{\alpha}_{1}, \bar{\alpha}_{2}\right)=(u(1-\sqrt{1-4 c / u}) / 2,(1-\sqrt{1-4 c / u}) / 2)$ - point $P$ in the right panel of Figure 5 - then there is a unique crossing of the acceptance function and $\alpha_{2}=c / u\left(1-\alpha_{1}\right)$, i.e. $M B_{21}=c$. Now, the acceptance function passes above $\left(\bar{\alpha}_{1}, \bar{\alpha}_{2}\right)$ if

$$
\psi\left(\bar{\alpha}_{1}, \underline{\sigma}_{1}, \underline{\sigma}_{2}\right) \geq \bar{\alpha}_{2} .
$$

This condition relates $\underline{\sigma}_{1}$ and $\underline{\sigma}_{2}$. Rewrite (14) using Theorem 1 as $\underline{\sigma}_{2} \leq \eta\left(\underline{\sigma}_{1}\right)<\underline{\sigma}_{1}$, requiring a large enough "wedge" between the standards of the two colleges.

To show that (14) implies a unique crossing, consider the secant of $\alpha_{2}=c / u\left(1-\alpha_{1}\right)$ (the curve $M B_{21}=c$ ). It has an increasing secant if and only if $\alpha_{1} \geq 1 / 2$. To see this, differentiate $\alpha_{2} / \alpha_{1}=c / u \alpha_{1}\left(1-\alpha_{1}\right)$ in $\alpha_{1}$. Notice also that $M B_{12}=c$ intersects the diagonal $\alpha_{2}=\alpha_{1}$ at the points $\left(\alpha_{1}^{\ell}, \alpha_{2}^{\ell}\right)=(1 / 2-\sqrt{1-c / 4 u} / 2,1 / 2 u-\sqrt{1-c / 4 u} / 2 u)$ and $\left(\alpha_{1}^{h}, \alpha_{2}^{h}\right)=(1 / 2+\sqrt{1-c / 4 u} / 2,1 / 2 u+\sqrt{1-c / 4 u} / 2 u)>(1 / 2,1 / 2 u)$.

Condition (14) gives $\psi\left(\alpha_{1}^{\ell}, \underline{\sigma}_{1}, \underline{\sigma}_{2}\right)>\alpha_{2}^{\ell}$. Since $\underline{\sigma}_{2}<\underline{\sigma}_{1}$, we have $\psi\left(\alpha_{1}, \underline{\sigma}_{1}, \underline{\sigma}_{2}\right) \geq \alpha_{2}$ for all $\alpha_{1}$. Thus, the acceptance function crosses $M B_{21}=c$ at or above $\left(\alpha_{1}^{h}, \alpha_{2}^{h}\right)$. And since $\alpha_{1}^{h}>1 / 2$, the secant of $M B_{21}=c$ must be increasing at any intersection with the acceptance function. Hence, there must be a single crossing point.

Proof of PART (b), Step 2. We now show that this single crossing property in $\alpha$ implies another in $x$ : If caliber $x$ applies to college 2 (i.e., if $2 \in S(x)$ ), then any caliber $y<x$ that applies somewhere also applies to college 2 (i.e., $2 \in S(y)$ if $S(y) \neq \varnothing$ ). Suppose not; i.e., assume that $S(y)=\{1\}$. Then there are two cases: $S(x)=\{2\}$ or $S(x)=\{1,2\}$. The first cannot occur, for by Theorem $1 \alpha_{2}(x) / \alpha_{1}(x)<\alpha_{2}(y) / \alpha_{1}(y)$, and thus $\alpha_{2}(x) u \geq \alpha_{1}(x)$ implies $\alpha_{2}(y) u>\alpha_{1}(y)$, contradicting $S(x)=\{2\}$. The second case 
is ruled out by the monotonicity of $M B_{21}$ given condition (14), as caliber $y$ has greater incentives than $x$ to apply to college 2 , and thus $S(y)=\{1\}$ cannot be optimal. Finally, $S(y)=\varnothing$ if $\alpha_{2}(y) u<c$ by (14), which happens for low calibers below a threshold.

\section{A.6 The Law of Demand}

Claim 1 (The Falling Demand Curve) If either college raises its admission standard, then its enrollment falls, and thus its rival's enrollment rises.

Proof Step 1: The Applicant pool at College 1 Shrinks. When $\underline{\sigma}_{1}$ increases, the acceptance relation shifts up by Theorem 1, and thus the above type sets change as well. Fix a caliber $x \in \mathcal{C}_{2}$ or $x \in \Phi$, so that $1 \notin S(x) .{ }^{14}$ We will show that $x$ continues to apply either to college 2 only or nowhere, and thus the pool of applicants at college 1 shrinks. If $x \in \mathcal{C}_{2}$, then $\alpha_{2}(x) u-c \geq 0$ and $\alpha_{2}(x) u \geq \alpha_{1}(x)$, and this continues to hold after the increase in $\underline{\sigma}_{1}$, since $\alpha(x)$ falls while $\alpha_{2}(x)$ is constant. And if $x \in \Phi$, then clearly caliber $x$ will continue to apply nowhere when $\underline{\sigma}_{1}$ increases.

Proof Step 2: The applicant pool at college 2 expands. Fix a caliber $x \in$ $\mathcal{C}_{2}$ or $x \in \mathcal{B}$, so that $2 \in S(x)$. It suffices to show that caliber $x$ continues to apply to college 2 when the admission standard at college 1 increases. If $x \in \mathcal{C}_{2}$, then $\alpha_{2}(x) u-c \geq$ 0 and $\alpha_{2}(x) u \geq \alpha_{1}(x)$; these inequalities continue to hold after $\underline{\sigma}_{1}$ rises, since $\alpha_{1}(x)$ falls while $\alpha_{2}(x)$ remains constant. And if $x \in \mathcal{B}$, then $M B_{21}=\left(1-\alpha_{1}(x)\right) \alpha_{2}(x) u$ rises in $\underline{\sigma}_{1}$, encouraging caliber $x$ to apply to college 2 . Thus, $x \notin \mathcal{C}_{1} \cup \Phi$. Since $x$ was arbitrary, it follows that the applicant pool at college $2, \mathcal{B} \cup \mathcal{C}_{2}$, expands when $\underline{\sigma}_{1}$ increases.

\section{A.7 Existence of a Stable Equilibrium}

Claim 2 (Existence) A stable equilibrium exists. College 1 fills its capacity. Also, there exists $\bar{\kappa}_{1}\left(\kappa_{2}, c\right)<1-\kappa_{2}$ satisfying $\lim _{c \rightarrow 0} \bar{\kappa}_{1}\left(\kappa_{2}, c\right)=1-\kappa_{2}$ such that if $\kappa_{1} \leq$ $\bar{\kappa}_{1}\left(\kappa_{2}, c\right)$, then college 2 also fills its capacity in any equilibrium. If $\kappa_{1}>\bar{\kappa}_{1}\left(\kappa_{2}, c\right)$, then college 2 has excess capacity in some equilibrium.

Proof: For some insight, we choose the capacity $\bar{\kappa}_{1}$ given $\kappa_{2}$ so that when college 2 has no standards, both colleges exactly fill their capacity. This borderline capacity is less than $1-\kappa_{2}$ since a positive mass of students - perversely, those with the highest

\footnotetext{
${ }^{14}$ With a slight abuse of notation, we let $\Phi$ denote the set of calibers that apply nowhere. The same symbol was previously used to denote the analogous set in $\alpha$-space.
} 


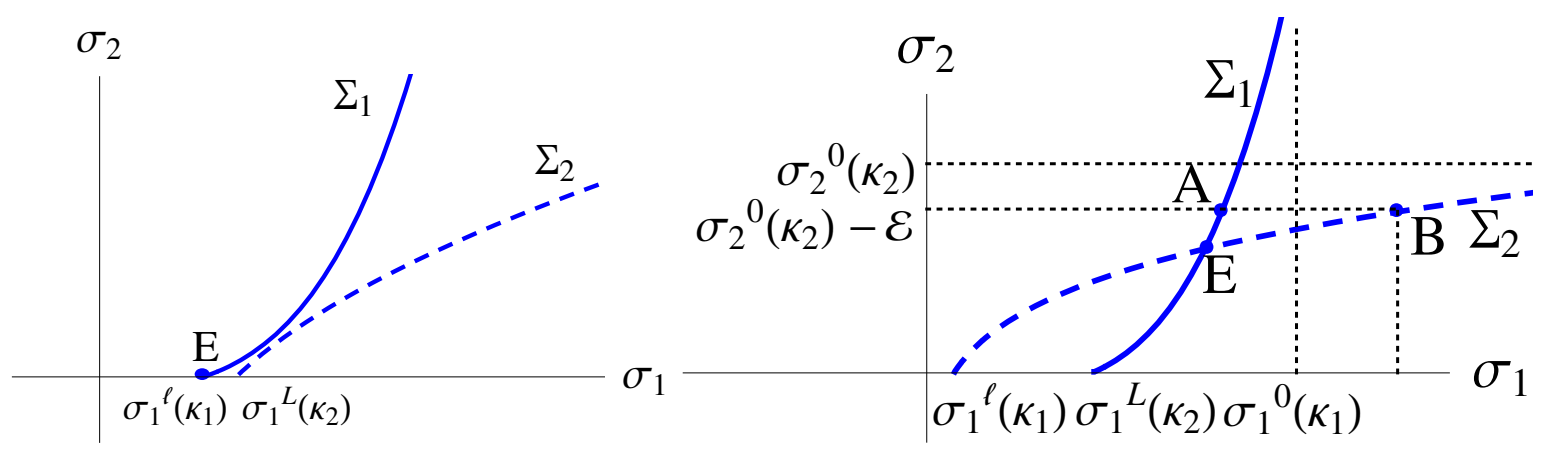

Figure 9: Equilibrium Existence. In the left panel, since $\kappa_{1}>\bar{\kappa}_{1}\left(\kappa_{2}\right)$, the best response functions $\Sigma_{1}$ and $\Sigma_{2}$ do not intersect, and equilibrium is at $E$ with $\underline{\sigma}_{2}=0$. The right panel depicts the proof of Claim 2 for the case $\kappa_{1}<\bar{\kappa}_{1}\left(\kappa_{2}\right)$.

calibers - applies just to college 1, and some are rejected. (This happens whenever one's admission chance at college 1 is at least $1-c / u$, by (1).)

For definiteness, we now denote the infimum signal by $-\infty$, and the supremum signal by $\infty$. Fix any $\kappa_{2} \in(0,1)$, and let $\underline{\sigma}_{1}^{l}\left(\kappa_{2}\right)$ be the unique solution to $\kappa_{2}=\mathcal{E}_{2}\left(\underline{\sigma}_{1},-\infty\right)$, i.e., when college 2 accepts everybody. (Existence and uniqueness of $\underline{\sigma}_{1}^{l}\left(\kappa_{2}\right)$ follows from $\mathcal{E}_{2}(-\infty,-\infty)=0, \mathcal{E}_{2}(\infty,-\infty)=1$, and $\mathcal{E}_{2}\left(\underline{\sigma}_{1},-\infty\right)$ increasing and continuous in $\left.\underline{\sigma}_{1}.\right)$

Define $\bar{\kappa}_{1}\left(\kappa_{2}\right)=\mathcal{E}_{1}\left(\underline{\sigma}_{1}^{l}\left(\kappa_{2}\right),-\infty\right)$. Let $\kappa_{1} \geq \bar{\kappa}_{1}\left(\kappa_{2}\right)$. We claim that there exists an equilibrium in which college 2 accepts everybody, and college 1 sets a threshold $\underline{\sigma}_{1}^{\ell}\left(\kappa_{1}\right)$, the unique solution to $\kappa_{1}=\mathcal{E}_{1}\left(\underline{\sigma}_{1},-\infty\right)$, which satisfies $\underline{\sigma}_{1}^{\ell}\left(\kappa_{1}\right) \leq \underline{\sigma}_{1}^{l}\left(\kappa_{2}\right)$. For since college 2 rejects no one, $\underline{\sigma}_{1}^{\ell}\left(\kappa_{1}\right)$ fills college 1's capacity exactly. The enrollment at college 2 is then $\mathcal{E}_{2}\left(\underline{\sigma}_{1}^{\ell}\left(\kappa_{1}\right),-\infty\right) \leq \kappa_{2}\left(\operatorname{as} \underline{\sigma}_{1}^{\ell}\left(\kappa_{1}\right) \leq \underline{\sigma}_{1}^{l}\left(\kappa_{2}\right)\right.$ and $\mathcal{E}_{2}\left(\underline{\sigma}_{1}, \underline{\sigma}_{2}\right)$ is increasing in $\left.\underline{\sigma}_{1}\right)$, so by accepting everybody college 2 fills as much capacity as it can. This equilibrium is trivially stable, as $\Sigma_{2}$ is 'flat' at the crossing point (see Figure 9, left panel). Moreover, if $\kappa_{1}>\bar{\kappa}_{1}\left(\kappa_{2}\right)$, then college 2 has excess capacity in this equilibrium.

Assume now $\kappa_{1}<\bar{\kappa}_{1}\left(\kappa_{2}\right)$. We will show that the continuous functions $\Sigma_{1}$ and $\Sigma_{2}$ must cross at least once (i.e., an equilibrium exists), and that the slope condition is met (i.e., it is stable). First, in this case $\underline{\sigma}_{1}^{l}\left(\kappa_{2}\right)<\underline{\sigma}_{1}^{\ell}\left(\kappa_{1}\right)$ or, equivalently, $\Sigma_{2}^{-1}\left(-\infty, \kappa_{2}\right)<$ $\Sigma_{1}\left(-\infty, \kappa_{1}\right)$. Second, as the standard of college 2 goes to infinity, college 1's threshold converges to $\underline{\sigma}_{1}^{u}\left(\kappa_{1}\right)<\infty$, the unique solution to $\kappa_{1}=\mathcal{E}_{1}\left(\underline{\sigma}_{1}, \infty\right)$. This is the largest threshold that college 1 can set given $\kappa_{1}$. Similarly, as the standard of college 1 goes to infinity, college 2's threshold converges to $\underline{\sigma}_{2}^{u}\left(\kappa_{2}\right)<\infty$, the unique solution to $\kappa_{2}=$ $\mathcal{E}_{2}\left(\infty, \underline{\sigma}_{2}\right)$, i.e. the largest threshold that college 2 can set given $\kappa_{2}$. Third, for $\epsilon>0$ small enough, the unique solution to $\kappa_{1}=\mathcal{E}_{1}\left(\underline{\sigma}_{1}, \underline{\sigma}_{2}^{u}\left(\kappa_{2}\right)-\epsilon\right)$ lies below the unique solution to 
$\kappa_{2}=\mathcal{E}_{2}\left(\underline{\sigma}_{1}, \underline{\sigma}_{2}^{u}\left(\kappa_{2}\right)-\epsilon\right)$. Equivalently, $\Sigma_{2}^{-1}\left(\underline{\sigma}_{2}^{u}\left(\kappa_{2}\right)-\epsilon, \kappa_{2}\right)>\Sigma_{1}\left(\underline{\sigma}_{2}^{u}\left(\kappa_{2}\right)-\epsilon, \kappa_{1}\right)$.

Since $\Sigma_{2}^{-1}\left(-\infty, \kappa_{2}\right)<\Sigma_{1}\left(-\infty, \kappa_{1}\right)$ and $\Sigma_{2}^{-1}\left(\underline{\sigma}_{2}^{u}\left(\kappa_{2}\right)-\epsilon, \kappa_{2}\right)>\Sigma_{1}\left(\underline{\sigma}_{2}^{u}\left(\kappa_{2}\right)-\epsilon, \kappa_{1}\right)$ (graphically, point $A$ is to the left of point $B$ in Figure 9), and $\Sigma_{1}$ and $\Sigma_{2}$ are continuous, by the Intermediate Value Theorem, they must cross at least once with the slope condition being satisfied (see Figure 9, right panel). Thus, a stable equilibrium exists when $\kappa_{1}<\bar{\kappa}_{1}\left(\kappa_{2}\right)$. Moreover, in any equilibrium there is no excess capacity at either college, since $\Sigma_{2}^{-1}\left(-\infty, \kappa_{2}\right)<\Sigma_{1}\left(-\infty, \kappa_{1}\right)$.

Hence, a stable equilibrium exists for any $\kappa_{2} \in(0,1)$. Capacities are exactly filled when $\kappa_{1} \leq \bar{\kappa}_{1}\left(\kappa_{2}\right)$, while there can be excess capacity at college 2 whenever $\kappa_{1}>\bar{\kappa}_{1}\left(\kappa_{2}\right)$.

Since $\kappa_{2}=\mathcal{E}_{1}\left(\underline{\sigma}_{1}^{l}\left(\kappa_{2}\right),-\infty\right), \bar{\kappa}_{1}\left(\kappa_{2}\right)$ equals $1-\kappa_{2}$ plus the mass of students who only applied to, and were rejected by, college 1 . This mass vanishes as $c$ vanishes, for then everybody applies to both colleges. So $\bar{\kappa}_{1}\left(\kappa_{2}\right)$ converges to $1-\kappa_{2}$ as $c$ goes to zero.

\section{A.8 Sorting Equilibrium Implies Stochastic Dominance of Types}

Claim 3 (Sorting and the Caliber Distribution) In any sorting equilibrium, the caliber distribution at college 1 first-order stochastically dominates that at college 2.

Proof: A monotone student strategy is represented by the partition of the set of types:

$$
\Phi=\left[0, \xi_{2}\right), \mathcal{C}_{2}=\left[\xi_{2}, \xi_{B}\right), \mathcal{B}=\left[\xi_{B}, \xi_{1}\right), \mathcal{C}_{1}=\left[\xi_{1}, \infty\right)
$$

where $\xi_{2}<\xi_{B}<\xi_{1}$ are defined by the intersection of the acceptance function with $c / u$, $\alpha_{2}=\left(1-c / \alpha_{1}\right) / u$ (i.e., $M B_{12}=c$ ), and $\alpha_{2}=c /\left[u\left(1-\alpha_{1}\right)\right]$ (i.e., $M B_{21}=c$ ), respectively.

Fix $\underline{\sigma}_{1}$ and $\underline{\sigma}_{2}$. Let $f_{1}(x)$ and $f_{2}(x)$ be the densities of calibers accepted at colleges 1 and 2, respectively. Formally,

$$
\begin{aligned}
f_{1}(x) & =\frac{\alpha_{1}(x) f(x)}{\int_{\xi_{B}}^{\infty} \alpha_{1}(t) f(t) d t} I_{\left[\xi_{B}, \infty\right)}(x) \\
f_{2}(x) & =\frac{I_{\left[\xi_{2}, \xi_{B}\right]}(x) \alpha_{2}(x) f(x)+\left(1-I_{\left[\xi_{2}, \xi_{B}\right]}(x)\right) \alpha_{2}(x)\left(1-\alpha_{1}(x)\right) f(x)}{\int_{\xi_{2}}^{\xi_{B}} \alpha_{2}(s) f(s) d s+\int_{\xi_{B}}^{\xi_{1}} \alpha_{2}(s)\left(1-\alpha_{1}(s)\right) f(s) d s} I_{\left[\xi_{2}, \xi_{1}\right]}(x),(17
\end{aligned}
$$

where $I_{A}$ is the indicator function of the set $A$.

We shall show that, if $x_{L}, x_{H} \in[0, \infty)$, with $x_{H}>x_{L}$, then $f_{1}\left(x_{H}\right) f_{2}\left(x_{L}\right) \geq$ $f_{2}\left(x_{H}\right) f_{1}\left(x_{L}\right)$; i.e., $f_{i}(x)$ is log-supermodular in $(-i, x)$, or it satisfies MLRP. The result follows as MLRP implies that the cdfs are ordered by first-order stochastic dominance. 
Using (16) and (17), $f_{1}\left(x_{H}\right) f_{2}\left(x_{L}\right) \geq f_{2}\left(x_{H}\right) f_{1}\left(x_{L}\right)$ is equivalent to

$$
\begin{aligned}
& \alpha_{1 H} I_{\left[\xi_{B}, \infty\right)}\left(x_{H}\right)\left(I_{\left[\xi_{2}, \xi_{B}\right]}\left(x_{L}\right) \alpha_{2 L}+\left(1-I_{\left[\xi_{2}, \xi_{B}\right]}\left(x_{L}\right)\right) \alpha_{2 L}\left(1-\alpha_{1 L}\right)\right) I_{\left[\xi_{2}, \xi_{1}\right]}\left(x_{L}\right) \geq \\
& \alpha_{1 L} I_{\left[\xi_{B}, \infty\right)}\left(x_{L}\right)\left(I_{\left[\xi_{2}, \xi_{B}\right]}\left(x_{H}\right) \alpha_{2 H}+\left(1-I_{\left[\xi_{2}, \xi_{B}\right]}\left(x_{H}\right)\right) \alpha_{2 H}\left(1-\alpha_{1 H}\right)\right) I_{\left[\xi_{2}, \xi_{1}\right]}\left(x_{H}\right),
\end{aligned}
$$

where $\alpha_{i j}=\alpha_{i}\left(x_{j}\right), i=1,2, j=L, H$. It is easy to show that the only nontrivial case is when $x_{L}, x_{H} \in\left[\xi_{B}, \xi_{1}\right]$ (in all the other cases, either both sides are zero, or only the right side is). If $x_{L}, x_{H} \in\left[\xi_{B}, \xi_{1}\right]$, then (18) becomes $\alpha_{1 H} \alpha_{2 L}\left(1-\alpha_{1 L}\right) \geq \alpha_{1 L} \alpha_{2 H}\left(1-\alpha_{1 H}\right)$, or

$$
\begin{aligned}
& \left(1-G\left(\underline{\sigma}_{1} \mid x_{H}\right)\right)\left(1-G\left(\underline{\sigma}_{2} \mid x_{L}\right)\right) G\left(\underline{\sigma}_{1} \mid x_{L}\right) \geq \\
& \left(1-G\left(\underline{\sigma}_{1} \mid x_{L}\right)\right)\left(1-G\left(\underline{\sigma}_{2} \mid x_{H}\right)\right) G\left(\underline{\sigma}_{1} \mid x_{H}\right) .
\end{aligned}
$$

Since $g(\sigma \mid x)$ satisfies MLRP, it follows that $G(\sigma \mid x)$ is decreasing in $x$, and hence $G\left(\underline{\sigma}_{1} \mid x_{L}\right) \geq G\left(\underline{\sigma}_{1} \mid x_{H}\right)$. Next, $1-G(\sigma \mid x)$ is log-supermodular in $(x, \sigma)$, and hence

$$
\left(1-G\left(\underline{\sigma}_{1} \mid x_{H}\right)\right)\left(1-G\left(\underline{\sigma}_{2} \mid x_{L}\right)\right) \geq\left(1-G\left(\underline{\sigma}_{1} \mid x_{L}\right)\right)\left(1-G\left(\underline{\sigma}_{2} \mid x_{H}\right)\right),
$$

as $\underline{\sigma}_{1}>\underline{\sigma}_{2}$ in a sorting equilibrium. Thus, (19) is satisfied, thereby proving that $f_{i}(x)$ is log-supermodular in $(-i, x)$, and so $F_{1}$ first-order stochastically dominates $F_{2}$.

\section{A.9 Changing Application Costs}

Claim 4 (College Comparative Statics) If the application costs at college 2 rises, then both admission standards fall. If the equilibrium is sorting, then the distribution of calibers of student enrolled at college 1 stochastically improves.

Proof: Assume a sorting equilibrium. We modify (15) for different costs: $\xi_{1}$ is defined by $M B_{21}=c_{2}, \xi_{B}$ by $M B_{12}=c_{1}$, and $\xi_{2}$ by $\alpha_{2} u=c_{2}$. If $c_{2}$ rises, then $\xi_{1}$ drops, $\xi_{2}$ rises, and $\xi_{B}$ is unchanged; thus, the applicant pool at college 2 shrinks, and at college 1 is unchanged. So the $\Sigma_{2}$ curve shifts down, while $\Sigma_{1}$ remains unchanged. The functions now cross at a lower threshold pair, and so both standards $\underline{\sigma}_{1}, \underline{\sigma}_{2}$ both fall.

Next consider an increase in $c_{1}$. This riases $\xi_{B}$, which shrinks the applicant pool at college 1 , and increases the enrollment at college 2 , at a fixed admission standard. This shifts $\Sigma_{1}$ left and $\Sigma_{2}$ up. While the effect on the standard $\underline{\sigma}_{2}$ is ambiguous, we now deduce that $\underline{\sigma}_{1}$ falls. Differentiating (4) and (5) with respect to $c_{1}$, and using Cramer's Rule:

$$
\frac{\partial \underline{\sigma}_{1}}{\partial c_{1}}=\frac{\left(\partial \mathcal{E}_{2} / \partial c_{1}\right)\left(\partial \mathcal{E}_{1} / \partial \underline{\sigma}_{2}\right)-\left(\partial \mathcal{E}_{1} / \partial c_{1}\right)\left(\partial \mathcal{E}_{2} / \partial \underline{\sigma}_{2}\right)}{\left(d \mathcal{E}_{1} / d \underline{\sigma}_{1}\right)\left(d \mathcal{E}_{2} / d \underline{\sigma}_{2}\right)-\left(d \mathcal{E}_{2} / d \underline{\sigma}_{1}\right)\left(d \mathcal{E}_{1} / d \underline{\sigma}_{2}\right)}
$$


Since the equilibrium is stable, the slope of $\Sigma_{1}$ is steeper that of $\Sigma_{2}$, and thus the denominator is positive. Let $P_{i}(\xi \mid y)$ be the portfolio density shift to college $i$ at type $\xi$ given an increment to standard or cost $y$, and let $S_{2}(A)$ be the own-standards effect at college 2 in set $A$. Then parse the enrollment derivatives into the portfolio and standards effects: $d \mathcal{E}_{1} / d c_{1}=P_{1}\left(\xi_{B} \mid c_{1}\right)<0, d \mathcal{E}_{2} / d \underline{\sigma}_{2}=\Sigma_{i=2, B, 1} P_{2}\left(\xi_{i} \mid \underline{\sigma}_{2}\right)-S_{2}\left(C_{2}\right)-S_{2}(B)<0$, $d \mathcal{E}_{2} / d c_{1}=P_{2}\left(\xi_{B} \mid c_{1}\right)>0$, and $d \mathcal{E}_{1} / d \underline{\sigma}_{2}=P_{1}\left(\xi_{B} \mid \underline{\sigma}_{2}\right)>0$. If $c_{1}$ slightly rises, then $\xi_{B}$ rises by some $\delta>0$. Thus, college 1 loses mass $f\left(\xi_{B}\right) \alpha_{1} \delta$ of students, and college 2 gains mass $f\left(\xi_{B}\right) \alpha_{1} \alpha_{2} \delta$ of students who would have gone to college 1 . Likewise, if $\underline{\sigma}_{2}$ slightly rises, then $\xi_{B}$ falls by some $\delta^{\prime}$, and college 1 gains mass $f\left(\xi_{B}\right) \alpha_{1} \delta^{\prime}$ and college 2 loses mass $f\left(\xi_{B}\right) \alpha_{1} \alpha_{2} \delta^{\prime}$. Thus, $P_{1}\left(\xi_{B} \mid \underline{\sigma}_{2}\right) P_{2}\left(\xi_{B} \mid c_{1}\right)-P_{1}\left(\xi_{B} \mid c_{1}\right) P_{2}\left(\xi_{B} \mid \underline{\sigma}_{2}\right)$ equals

$$
\left[f\left(\xi_{B}\right) \alpha_{1} \delta^{\prime}\right]\left[f\left(\xi_{B}\right) \alpha_{1} \alpha_{2} \delta\right]-\left[f\left(\xi_{B}\right) \alpha_{1} \delta\right]\left[f\left(\xi_{B}\right) \alpha_{1} \alpha_{2} \delta^{\prime}\right]=0
$$

Hence, the numerator in (20) reduces to

$$
-P_{1}\left(\xi_{B} \mid c_{1}\right)\left[P_{2}\left(\xi_{2} \mid \underline{\sigma}_{2}\right)+P\left(\xi_{1} \mid \underline{\sigma}_{2}\right)-S_{2}\left(\mathcal{C}_{2}\right)-S_{2}(\mathcal{B})\right]<0
$$

In a sorting equilibrium, the applicant pool at college 1 consists of calibers $x \in$ $\left[\xi_{B}, \infty\right)$. From the last part, any cost increase depresses $\underline{\sigma}_{1}$ in equilibrium. It follows that $\xi_{B}$ rises in equilibrium - since college 1 has the same capacity as before, if it is to have lower standards, it must also have fewer applicants. Let $\left(\xi_{B}^{0}, \underline{\sigma}_{1}^{0}\right)$ be the old equilibrium pair and $\left(\xi_{B}^{1}, \underline{\sigma}_{1}^{1}\right)$ the new one, with $\xi_{B}^{0}<\xi_{B}^{1}$ and $\underline{\sigma}_{1}^{0}>\underline{\sigma}_{1}^{1}$. Then the distribution function of enrolled students at college 1 under equilibrium $i=0,1$ is:

$$
F_{1}^{i}(x)=\frac{\int_{\xi_{B}^{i}}^{x}\left(1-G\left(\underline{\sigma}_{1}^{i} \mid t\right)\right) f(t) d t}{\int_{\xi_{B}^{i}}^{\infty}\left(1-G\left(\underline{\sigma}_{1}^{i} \mid t\right)\right) f(t) d t}
$$

We must show $F_{1}^{1}(x) \leq F_{1}^{0}(x)$ for all $x \in\left[\xi_{B}^{1}, \infty\right)$. For any $x$, the denominators on both sides equal $k_{1}$, so cancel them. Now notice that $0=F_{1}^{1}\left(\xi_{B}^{1}\right)<F_{1}^{0}\left(\xi_{B}^{1}\right)$ and $\lim _{x \rightarrow \infty} F_{1}^{1}(x)=\lim _{x \rightarrow \infty} F_{1}^{0}(x)=1$. Since both functions are continuous in $x$, if $\partial F_{1}^{1} / \partial x>\partial F_{1}^{0} / \partial x$ for all $x \in\left[\xi_{B}^{1}, \infty\right)$, then $F_{1}^{1}(x)<F_{1}^{0}(x)$. But this requires $\left(1-G\left(\underline{\sigma}_{1}^{1} \mid x\right)\right) f(x)>\left(1-G\left(\underline{\sigma}_{1}^{0} \mid x\right)\right) f(x)$, which follows from $\underline{\sigma}_{1}^{1}<\underline{\sigma}_{1}^{0}$. 


\section{A.10 Sorting and Non-Sorting: Proof of Theorem 2}

Part (a): College 2 IS Too Good. We prove that there exists $\bar{\kappa}_{2}\left(\kappa_{1}\right)>0$ so that college 2 sets a higher admissions standard than college 1 in a stable equilibrium, for any capacity $\kappa_{2} \leq \bar{\kappa}_{2}\left(\kappa_{1}\right)$. Fix any $\kappa_{1}, \kappa_{2}$, such that $\kappa_{1}+\kappa_{2}<1$. We shall proceed in two steps. First, we show that since $u>0.5$, we can use Theorem 1 to construct a non-sorting equilibrium in which colleges' behavior is monotone but students' is not. Second, we show that all equilibria induce the same type of behavior.

Step 1: Towards an Acceptance Function. When $u>0.5$, the secant from the origin to $M B_{12}=c$ falls as $\alpha_{1}$ tends to $c /(1-u)$ - as in the left panel of Figure 5 . So for some $\underline{z}<c /(1-u) \mathrm{s}$, a line from the origin to $(\underline{z}, 1)$ slices the $M B_{12}$ curve twice. This would imply non-monotone student behavior if that line belonged to the acceptance function, such as: $h:[0,1] \rightarrow[0,1]$ by $h(\alpha)=\alpha / \underline{z}$ and on $[0, \underline{z})$, and $h\left(\alpha_{1}\right)=1$ for $\alpha_{1} \geq \underline{z}$.

Step 2: A PieCEWise-linear ACCEPtance CHAnCE $\alpha_{1}$. Choose $\underline{\xi}$ and $\bar{\xi}$ that uniquely solve $\kappa_{1}=\int_{\bar{\xi}}^{\infty} f(x) d x$ and $\kappa_{2}=\int_{\underline{\xi}}^{\bar{\xi}} f(x) d x$. Set $\alpha_{1}(x)=0$ for $x<\underline{\xi}$. This function then jumps up to the rising line segment $\alpha_{1}(x)=\omega(x) \underline{z}+(1-\omega(x)) c /(1-u)$ for $x \in[\underline{\xi}, \bar{\xi})$, where $\omega(x) \equiv(\bar{\xi}-x) /(\bar{\xi}-\underline{\xi})$. Lastly, $\alpha_{1}$ jumps up $\alpha_{1}(x)=1$ for $x>\bar{\xi}$.

Step 3: Student Behavior. Observe that $h(0)=0$ and $h(1)=1$, and that $h$ is weakly increasing, with both $h(\alpha) / \alpha$ and $[1-h(\alpha)] /[1-\alpha]$ weakly decreasing. In this sense, $h$ is a weakly regular function. This suggests that we set $\alpha_{2}(x) \equiv h\left(\alpha_{1}(x)\right)$.

In this case, students $x \in[0, \underline{\xi})$ are accepted with zero chance at either college, and so apply nowhere. Next, because $h(\underline{z})=1$, any calibers $x \in[\underline{\xi}, \bar{\xi})$ are accepted with chance one at college 2 , and with chance between $\underline{z}$ and $c /(1-u)$ at college 1 . Further, any student $\bar{\xi}$ strictly prefers just to apply to college 2 (as in Figure 5). To see this, observe that $M B_{12}=(c /(1-u))\left(1-\alpha_{2} u\right)>(c /(1-u))(1-u)=c$ when $\alpha_{2}=c /(1-u)$ and $\alpha_{1}=1$. Lastly, calibers $x>\bar{\xi}$ are always accepted at college 1 and only apply there.

Step 4: Smoothing the Construction. By smoothly bending the function $h$ inside $(0,1)$, an arbitrarily close function $h^{*}$ is also regular. Next, we create a continuous and smooth acceptance chance $\tilde{\alpha}$. Any four small enough numbers, $\underline{\varepsilon}, \underline{\epsilon}, \bar{\varepsilon}, \bar{\epsilon}>0$, yield a unique Bezier approximation $\tilde{\alpha}$ tangent to $\alpha$ at the four points $\underline{\xi}-\underline{\varepsilon}, \underline{\xi}+\underline{\epsilon}, \bar{\xi}-\bar{\varepsilon}, \bar{\xi}+\bar{\epsilon}$. Then $\tilde{\alpha}_{1}$ - and so the enrollment at college 1 - falls in $\bar{\epsilon}$, and rises in $\bar{\varepsilon}$. Also, $\tilde{\alpha}_{2}=h^{*}\left(\tilde{\alpha}_{1}\right)$ falls in $\bar{\epsilon}$, and rises in $\bar{\varepsilon}$, and it also falls in $\underline{\epsilon}$, and rises in $\underline{\varepsilon}$. Enrollment at college 2 shares this monotonicity, but the enrollment at college 1 is unaffected by $\underline{\epsilon}$ and $\underline{\varepsilon}$.

Fix a small $\bar{\epsilon}>0$. Choose $\bar{\varepsilon}>0$ so that college 1 still fills its capacity. WLOG, 

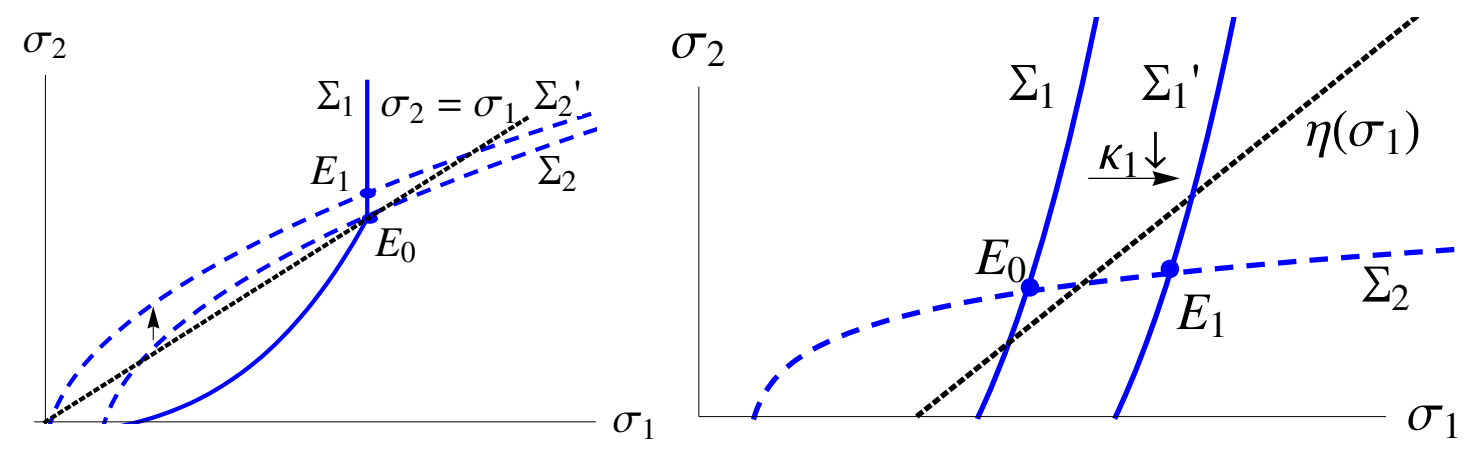

Figure 10: Existence of Sorting and Non-Sorting Equilibria In the left panel, we depict the non-sorting equilibrium constructed in the proof of Theorem 2. As $\kappa_{2}$ decreases, $\Sigma_{2}$ shifts up, leading to a non-sorting equilibrium at $\mathcal{E}_{1}$. The right panel illustrates the proof of Theorem 2. As $\kappa_{1}$ falls, the equilibrium standards at $\mathcal{E}_{1}$ are guaranteed to satisfy $\underline{\sigma}_{2}<\eta\left(\underline{\sigma}_{1}\right)$, thereby obtaining a sorting equilibrium.

enrollment at college 2 has fallen. Then choose $\underline{\varepsilon}>0$ large enough so that college 2 is over its capacity, then for some $\underline{\epsilon}>0$, the former enrollment at college 2 is restored.

Theorem 1 now yields a signal density $g(\sigma \mid x)$ and thresholds $\underline{\sigma}_{1}>\underline{\sigma}_{2}$ such that $h^{*}$ is the acceptance function. We have thus constructed a non-sorting equilibrium.

Part (b): College 2 is Too Small. The proof is constructive, exploiting our graphical analysis. To begin, consider the point $\left(\alpha_{1}, \alpha_{2}\right)=(c, c / u)$ on the line $\alpha_{2}=\alpha_{1} / u$. Then the acceptance function evaluated at $\alpha_{1}=c$ is below $c / u$ if and only if

$$
\psi\left(c, \underline{\sigma}_{1}, \underline{\sigma}_{2}\right)<c / u
$$

We will restrict attention to pairs $\left(\underline{\sigma}_{1}, \underline{\sigma}_{2}\right)$ such that (21) holds. In this case, any student who applies to college starts by adding college 1 to his portfolio, and this happens as soon as $\alpha_{1}(x) \geq c$, or when $x \geq \xi\left(c, \underline{\sigma}_{1}\right)$. Then enrollment at college 1 is given by

$$
\mathcal{E}_{1}\left(\underline{\sigma}_{1}, \underline{\sigma}_{2}\right)=\int_{\xi\left(c, \underline{\sigma}_{1}\right)}^{\infty}\left(1-G\left(\underline{\sigma}_{1} \mid x\right)\right) f(x) d x
$$

which is independent of $\underline{\sigma}_{2}$. Thus, for any capacity $\kappa_{1} \in(0,1)$, a unique threshold $\underline{\sigma}_{1}\left(\kappa_{1}\right)$ solves $\kappa_{1}=\mathcal{E}_{1}\left(\underline{\sigma}_{1}, \underline{\sigma}_{2}\right)$. (The $\Sigma_{1}^{-1}$ function is "vertical" when (21) holds, since the applicant pool at college 1 does not depend on college 2's admissions threshold.)

The analysis above allows us to restrict attention to finding equilibria within the set of thresholds $\left(\underline{\sigma}_{1}, \underline{\sigma}_{2}\right)$ such that $\underline{\sigma}_{1}=\underline{\sigma}_{1}\left(\kappa_{1}\right)$ and $\underline{\sigma}_{2}$ satisfies $\psi\left(c, \underline{\sigma}_{1}\left(\kappa_{1}\right), \underline{\sigma}_{2}\right)<c / u$. 
Enrollment at college 2 is given by

$$
\mathcal{E}_{2}\left(\underline{\sigma}_{1}\left(\kappa_{1}\right), \underline{\sigma}_{2}\right)=\int_{B} G\left(\underline{\sigma}_{1}\left(\kappa_{1}\right) \mid x\right)\left(1-G\left(\underline{\sigma}_{2} \mid x\right)\right) f(x) d x
$$

which is continuous, decreasing in $\underline{\sigma}_{2}$, and increasing in $\underline{\sigma}_{1}$ (see Claim 1). Thus, $\kappa_{2}=$ $\mathcal{E}_{2}\left(\underline{\sigma}_{1}\left(\kappa_{1}\right), \underline{\sigma}_{2}\right)$ yields $\underline{\sigma}_{2}=\Sigma_{2}\left(\underline{\sigma}_{1}\left(\kappa_{1}\right), \kappa_{2}\right)$, which is strictly decreasing in $\kappa_{2}$.

Given $\kappa_{1}$, let $\bar{\kappa}_{2}\left(\kappa_{1}\right)=E_{2}\left(\underline{\sigma}_{1}\left(\kappa_{1}\right), \underline{\sigma}_{1}\left(\kappa_{1}\right)\right)$ be the level of college 2 capacity so that equilibrium ensues if both colleges set the same threshold. ${ }^{15}$ Since $\Sigma_{2}$ strictly falls in $\kappa_{2}$, for any $\kappa_{2}<\bar{\kappa}_{2}\left(\kappa_{1}\right)$, an equilibrium exists with $\underline{\sigma}_{2}>\underline{\sigma}_{1}\left(\kappa_{1}\right)$. Then $(a)$ for any $\kappa_{1} \in(0,1)$ and $\kappa_{2} \in\left(0, \bar{\kappa}_{2}\left(\kappa_{1}\right)\right]$, there is a unique equilibrium with $\underline{\sigma}_{1}=\underline{\sigma}_{1}\left(\kappa_{1}\right)$ and $\underline{\sigma}_{2} \geq \underline{\sigma}_{1}\left(\kappa_{1}\right)$, having $(b)$ non-monotone college and student behavior (Figure 10, left). ${ }^{16}$

Part $(c)$ : Conditions for Equilibrium Sorting. We prove that there exists $\underline{\kappa}_{1}\left(\kappa_{2}\right)>0$ such that if $\kappa_{1} \leq \underline{\kappa}_{1}\left(\kappa_{2}\right)$ and $u \leq 0.5$, then there are only sorting equilibria and neither college has excess capacity.

Fix $\kappa_{2} \in(0,1)$. We first show that the stable equilibrium with no excess capacity derived in Claim 2 is also sorting when the capacity of college 1 is small enough. More precisely, there is a threshold $\underline{\kappa}_{1}\left(\kappa_{2}\right)$, smaller than the bound $\bar{\kappa}_{1}\left(\kappa_{2}\right)$ defined in the proof of Claim 2, such that for all $\kappa_{1} \in\left(0, \underline{\kappa}_{1}\left(\kappa_{2}\right)\right)$, there is a pair of admissions thresholds $\left(\underline{\sigma}_{1}, \underline{\sigma}_{2}\right)$ that satisfies $\kappa_{1}=\mathcal{E}_{1}\left(\underline{\sigma}_{1}, \underline{\sigma}_{2}\right), \kappa_{2}=\mathcal{E}_{2}\left(\underline{\sigma}_{1}, \underline{\sigma}_{2}\right)$, and $\underline{\sigma}_{2}<\eta\left(\underline{\sigma}_{1}\right)$ (i.e., a sorting equilibrium), and $\partial \Sigma_{1} / \partial \underline{\sigma}_{2} \partial \Sigma_{2} / \partial \underline{\sigma}_{1}<1$ (i.e., the equilibrium is stable).

The proof uses three easily-verified properties of the function $\eta$ : $(a) \eta$ is strictly increasing; (b) $\underline{\sigma}_{2}=\eta\left(\underline{\sigma}_{1}\right) \rightarrow \infty$ as $\underline{\sigma}_{1} \rightarrow \infty ;(c) \underline{\sigma}_{1}=\eta^{-1}\left(\underline{\sigma}_{2}\right) \rightarrow-\infty$ as $\underline{\sigma}_{2} \rightarrow-\infty$.

For any $\kappa_{1} \in\left(0, \bar{\kappa}_{1}\left(\kappa_{2}\right)\right)$, we know from Claim 2 that there exists a pair $\left(\underline{\sigma}_{1}, \underline{\sigma}_{2}\right)$ that satisfies $\kappa_{1}=\mathcal{E}_{1}\left(\underline{\sigma}_{1}, \underline{\sigma}_{2}\right)$ and $\kappa_{2}=\mathcal{E}_{2}\left(\underline{\sigma}_{1}, \underline{\sigma}_{2}\right)$, with $\left(\partial \Sigma_{1} / \partial \underline{\sigma}_{2}\right)\left(\partial \Sigma_{2} / \partial \underline{\sigma}_{1}\right)<1$.

\section{Claim 5 The pair $\left(\underline{\sigma}_{1}, \underline{\sigma}_{2}\right)$ is a sorting equilibrium when $\kappa_{1}$ is sufficiently small.}

Proof: Let $M\left(\kappa_{2}\right)=\left\{\left(\underline{\sigma}_{1}, \underline{\sigma}_{2}\right) \mid \kappa_{2}=\mathcal{E}_{2}\left(\underline{\sigma}_{1}, \underline{\sigma}_{2}\right)\right.$ and $\left.\underline{\sigma}_{2}=\eta\left(\underline{\sigma}_{1}\right)\right\}$. Graphically, this is the set of all pairs at which $\underline{\sigma}_{2}=\Sigma_{2}\left(\underline{\sigma}_{1}, \kappa_{2}\right)$ crosses $\underline{\sigma}_{2}=\eta\left(\underline{\sigma}_{1}\right)$.

If $M\left(\kappa_{2}\right)=\varnothing$ we are done, for then $\underline{\sigma}_{2}=\Sigma_{2}\left(\underline{\sigma}_{1}, \kappa_{2}\right)<\eta\left(\underline{\sigma}_{1}\right)$ for all $\underline{\sigma}_{1}$, including those at which $\kappa_{1}=\mathcal{E}_{1}\left(\underline{\sigma}_{1}, \underline{\sigma}_{2}\right)$ and $\kappa_{2}=E_{2}\left(\underline{\sigma}_{1}, \underline{\sigma}_{2}\right)$. To see this, note that $(i) \underline{\sigma}_{1}=$ $\eta^{-1}\left(\underline{\sigma}_{2}\right) \rightarrow-\infty$ as $\underline{\sigma}_{2} \rightarrow-\infty$, while we proved in Claim 2 that $\underline{\sigma}_{1}=\Sigma_{2}^{-1}\left(\underline{\sigma}_{2}, \kappa_{2}\right)$ converges to $\underline{\sigma}_{1}^{l}\left(\kappa_{2}\right)>-\infty$. Also, $(i i) \underline{\sigma}_{2}=\eta\left(\underline{\sigma}_{1}\right) \rightarrow \infty$ as $\underline{\sigma}_{1} \rightarrow \infty$, while we proved in

\footnotetext{
${ }^{15}$ It is not difficult to show that $\psi\left(c, \underline{\sigma}_{1}, \underline{\sigma}_{2}\right)<c / u$ is satisfied if $\underline{\sigma}_{2} \geq \underline{\sigma}_{1}\left(\kappa_{1}\right)$.

${ }^{16}$ We are not ruling out the existence of another equilibrium that does not satisfy (21).
} 
Claim 2 that $\underline{\sigma}_{2}=\Sigma_{2}\left(\underline{\sigma}_{1}, \kappa_{2}\right)$ converges to $\underline{\sigma}_{2}^{u}\left(\kappa_{2}\right)<\infty$. Properties $(i)$ and $(i i)$ reveal that if $\Sigma_{2}$ and $\eta$ do not intersect, then $\Sigma_{2}$ is everywhere below $\eta$.

If $M\left(\kappa_{2}\right) \neq \varnothing$, let $\left(\underline{\sigma}_{1}^{s}\left(\kappa_{2}\right), \underline{\sigma}_{2}^{s}\left(\kappa_{2}\right)\right)=\sup M\left(\kappa_{2}\right)$, which is finite by property $(b)$ of $\eta\left(\underline{\sigma}_{1}\right)$ and since $\underline{\sigma}_{2}=\Sigma_{2}\left(\underline{\sigma}_{1}, \kappa_{2}\right)$ converges to $\underline{\sigma}_{2}^{u}\left(\kappa_{2}\right)<\infty$ as $\underline{\sigma}_{1}$ goes to infinity (see the proof of Claim 2). Now, as $\kappa_{1}$ goes to zero, $\underline{\sigma}_{1}=\Sigma_{1}\left(\underline{\sigma}_{2}, \kappa_{1}\right)$ goes to infinity for any value of $\underline{\sigma}_{2}$, for college 1 becomes increasingly more selective to fill its dwindling capacity. Since $\underline{\sigma}_{2}$ is bounded above by $\underline{\sigma}_{2}^{u}\left(\kappa_{2}\right)$, there exists a threshold $\underline{\kappa}_{1}\left(\kappa_{2}\right) \leq \bar{\kappa}_{1}\left(\kappa_{2}\right)$ such that, for all $\kappa_{1} \in\left(0, \underline{\kappa}_{1}\left(\kappa_{2}\right)\right)$, the aforementioned pair $\left(\underline{\sigma}_{1}, \underline{\sigma}_{2}\right)$ that satisfies $\kappa_{1}=\mathcal{E}_{1}\left(\underline{\sigma}_{1}, \underline{\sigma}_{2}\right)$ and $\kappa_{2}=E_{2}\left(\underline{\sigma}_{1}, \underline{\sigma}_{2}\right)$ is strictly bigger than $\left(\underline{\sigma}_{1}^{s}\left(\kappa_{2}\right), \underline{\sigma}_{2}^{s}\left(\kappa_{2}\right)\right)$, thereby showing that it also satisfies $\underline{\sigma}_{2}<\eta\left(\underline{\sigma}_{1}\right)$. Hence, a sorting stable equilibrium exists for any $\kappa_{2}$ and $\kappa_{1} \in\left(0, \underline{\kappa}_{1}\left(\kappa_{2}\right)\right)$, with both colleges filling their capacities (see Figure 10, right panel).

To finish the proof, notice that, if there are multiple equilibria, both colleges fill their capacity in all of them (graphically, the conditions on capacities ensure that $\Sigma_{2}$ starts above $\Sigma_{1}$ for low values of $\underline{\sigma}_{1}$ and eventually ends below it). Moreover, adjusting the bound $\underline{\kappa}_{1}\left(\kappa_{2}\right)$ downward if needed, all equilibria are sorting (graphically, for $\kappa_{1}$ sufficiently small, the set of pairs at which $\Sigma_{1}$ and $\Sigma_{2}$ intersect are all below $\eta$ ).

\section{A.11 Affirmative Action: Negligible Feedback Effects}

We show that in a neighborhood of $\Delta_{1}=\Delta_{2}=0$, changes in $\Delta_{i}$ will have a negligible impact on $\underline{\sigma}_{j}, i, j=1,2$ at any stable solution solution to the capacity equations (6). (For this result alone, we also assume that the signal cdf derivative $G_{x}$ is continuous.) Given any discount pair $\left(\Delta_{1}, \Delta_{2}\right)$, the capacity equations with two groups are:

$$
\begin{aligned}
& \kappa_{1}=\phi \mathcal{E}_{1}^{\tau}\left(\underline{\sigma}_{1}-\Delta_{1}, \underline{\sigma}_{2}-\Delta_{2}\right)+(1-\phi) \mathcal{E}_{1}^{N}\left(\underline{\sigma}_{1}, \underline{\sigma}_{2}\right) \\
& \kappa_{2}=\phi \mathcal{E}_{2}^{\tau}\left(\underline{\sigma}_{1}-\Delta_{1}, \underline{\sigma}_{2}-\Delta_{2}\right)+(1-\phi) \mathcal{E}_{2}^{N}\left(\underline{\sigma}_{1}, \underline{\sigma}_{2}\right),
\end{aligned}
$$

where $\mathcal{E}_{i}^{\tau}, \mathcal{E}_{i}^{N}$ are the respective fractions of targeted and non-targeted groups enrolled at college $i$, defined just as in (4) and (5), for the sets of signals (15). Since the signal density $g=G_{\sigma}$ and its derivative $G_{x}$ are both continuous, all derivatives of the enrollment function (using Leibnitz rule) are continuous too. 
Differentiating equations $(22)$ and $(23)$ with respect to $\Delta_{1}$ :

$$
\begin{aligned}
\frac{J}{\phi} \frac{\partial \underline{\sigma}_{1}}{\partial \Delta_{1}} & =\sum_{i=1,2}(-1)^{i+1} \frac{\partial \mathcal{E}_{i}^{\tau}}{\partial\left(\underline{\sigma}_{1}-\Delta_{1}\right)}\left(\phi \frac{\partial \mathcal{E}_{3-i}^{\tau}}{\partial\left(\underline{\sigma}_{2}-\Delta_{2}\right)}+(1-\phi) \frac{\partial \mathcal{E}_{3-i}^{N}}{\partial \underline{\sigma}_{2}}\right) \\
\frac{J}{\phi} \frac{\partial \underline{\sigma}_{2}}{\partial \Delta_{1}} & =\sum_{i=1,2}(-1)^{i} \frac{\partial \mathcal{E}_{i}^{\tau}}{\partial\left(\underline{\sigma}_{1}-\Delta_{1}\right)}\left(\phi \frac{\partial \mathcal{E}_{3-i}^{\tau}}{\partial\left(\underline{\sigma}_{1}-\Delta_{1}\right)}+(1-\phi) \frac{\partial \mathcal{E}_{3-i}^{N}}{\partial \underline{\sigma}_{1}}\right)
\end{aligned}
$$

where the denominator, from Cramer's Rule, equals

$$
\begin{aligned}
J= & \left(\phi \frac{\partial \mathcal{E}_{1}^{\tau}}{\partial\left(\underline{\sigma}_{1}-\Delta_{1}\right)}+(1-\phi) \frac{\partial \mathcal{E}_{1}^{N}}{\partial \underline{\sigma}_{1}}\right)\left(\phi \frac{\partial \mathcal{E}_{2}^{\tau}}{\partial\left(\underline{\sigma}_{2}-\Delta_{2}\right)}+(1-\phi) \frac{\partial \mathcal{E}_{2}^{N}}{\partial \underline{\sigma}_{2}}\right) \\
& -\left(\phi \frac{\partial \mathcal{E}_{1}^{\tau}}{\partial\left(\underline{\sigma}_{2}-\Delta_{2}\right)}+(1-\phi) \frac{\partial \mathcal{E}_{1}^{N}}{\partial \underline{\sigma}_{2}}\right)\left(\phi \frac{\partial \mathcal{E}_{2}^{\tau}}{\partial\left(\underline{\sigma}_{1}-\Delta_{1}\right)}+(1-\phi) \frac{\partial \mathcal{E}_{2}^{N}}{\partial \underline{\sigma}_{1}}\right)
\end{aligned}
$$

is positive in any stable equilibrium - i.e. the two group version of the condition that the slope of $\Sigma_{1}$ exceed the slope of $\Sigma_{2}$ in $\S 4$ and $\S A .9$. Now, $\partial \underline{\sigma}_{1} / \partial \Delta_{1}=\phi>0$ and $\partial \underline{\sigma}_{2} / \partial \Delta_{1}=0$ when $\Delta_{1}=\Delta_{2}=0$, because the derivatives of the function $\mathcal{E}_{i}^{\tau}$, $\mathcal{E}_{i}^{N}$ at

colleges $i=1,2$ coincide. Thus, the feedback effects vanish when $\Delta_{1}=\Delta_{2}=0$, and are negligible in a neighborhood of it, by continuity of the enrollment derivatives. The analysis of the derivatives of $\underline{\sigma}_{i}, i=1,2$, with respect to $\Delta_{2}$ is analogous.

\section{A.12 Student Poaching: Proof of Theorem 4}

For simplicity, assuming that the infimum signal is $\sigma=0$ - which is WLOG by a simple monotone transformation of the signal. Let $\left(\Delta_{2}^{*}, \underline{\sigma}_{1}^{*}, \underline{\sigma}_{2}^{*}\right)$ solve the capacity equations (6) as well as $\underline{\sigma}_{2}^{*}=\Delta_{2}^{*}>0$. In other words, college 2 admits all early applicants.

Fix $\left(\underline{\sigma}_{1}^{*}, \underline{\sigma}_{2}^{*}\right)$. Given $\Delta_{2}$, let $V_{2}\left(\Delta_{2}\right)$ be the difference between early and regular shadow values, namely, the LHS minus the RHS of equation (10).

If $V_{2}\left(\Delta_{2}^{*}\right) \geq 0$, then $\left(\Delta_{2}^{*}, \underline{\sigma}_{1}^{*}, \underline{\sigma}_{2}^{*}\right)$ is an equilibrium where early applicants are favored, since $\Delta_{2}^{*}>0$. For each college fills its capacity, and either $V_{2}\left(\Delta_{2}^{*}\right)=0$, or $V_{2}\left(\Delta_{2}^{*}\right)>0$ which is a maximum for college 2 , since it admits all early applicants.

Assume instead that $V_{2}\left(\Delta_{2}^{*}\right)<0$. We will show that for some intermediate discount $\hat{\Delta}_{2} \in\left(0, \Delta_{2}^{*}\right)$, college 2 favors its early applicants in some equilibrium.

Lemma 2 The shadow value difference is positive when $\Delta_{2}=0$, or $V_{2}(0)>0$.

Proof: The expected payoff for the lowest caliber that applies early or regular to college 2 equals the application cost. Also, $M B_{21}=c$ for the highest caliber who applies early or 
regular to college 2 (see Figure 8). Altogether, the same set of students apply early and regular to college 2 coincide when it sets the same standards for both groups.

Next, recalling the discussion around (11), a larger fraction of the early than the regular group accept college 2 if admitted. Thus, college 2 suffers from less of an acceptance curse in the early than in the regular period. Thus, the shadow value of early students exceeds that of regular students, proving the Lemma.

Now, since the caliber and signal densities are continuous, $V_{2}\left(\Delta_{2}\right)$ is also continuous in $\Delta_{2}$. So by the Intermediate Value Theorem, there exists $\hat{\Delta}_{2} \in\left(0, \Delta_{2}^{*}\right)$ with $V_{2}\left(\hat{\Delta}_{2}\right)=0$. Thus, $\left(\Delta_{2}^{*}, \underline{\sigma}_{1}\left(\Delta_{2}^{*}\right), \underline{\sigma}_{2}\left(\Delta_{2}^{*}\right)\right)$ is an equilibrium where early applicants are favored.

\section{References}

Albrecht, J., P. Gautier, and S. Vroman (2003): "Equilibrium Directed Search with Multiple Applications," Tinbergen Institute Discussion Papers.

Anderson, A., And L. Smith (2007): "Assortative Matching and Reputation," University of Michigan mimeo.

Avery, C., A. Fairbanks, and R. Zeckhauser (2003): The Early Admissions Game: Joining the Elite. Harvard University Press.

Avery, C., And J. Levin (2009): "Early Admissions at Selective Colleges," forthcoming in American Economic Review.

Burdett, K., And K. Judd (1983): "Equilibrium Price Dispersion," Econometrica, 51(4), 955-69.

Burdett, K., S. Shi, and R. Wright (2001): "Pricing and Matching with Frictions," Journal of Political Economy, 109(5), 1060-1085.

Chade, H. (2006): "Matching with noise and the acceptance curse," Journal of Economic Theory, 129(1), 81-113.

Chade, H., and L. Smith (2006): "Simultaneous Search," Econometrica, 74(5), 12931307.

Fu, C. (2009): "Equilibrium Tuition, Applications, Admissions and Enrollment in the College Market," Working Paper, University of Pennsylvania. 
HoxBy, C. M. (2009): "The Changing Selectivity of American Colleges," NBER Working Paper No. 15446, October.

Karlin, S. (1968): Total Positivity, vol. 1. Stanford University Press, Stanford, CA.

Karlin, S., And Y. RinotT (1980): "Classes of orderings of measures and related correlation inequalities. I. Multivariate totally positive distributions," Journal of Multivariate Analysis, 10, 467-498.

Kircher, P., And M. Galenianos (2006): "Directed Search with Multiple Job Applications," mimeo, University of Pennsylvania.

LeE, S.-H. (2009): "Jumping the Curse: Early Contracting with Private Information in University Admissions," International Economic Review, 50(1), 1-38.

NAGYPÁL, E. (2004): "Optimal Application Behavior with Incomplete Information," mimeo, Northwestern University.

Shimer, R., And L. Smith (2000): "Assortative Matching and Search," Econometrica, 68(2), 343-369.

Smith, L. (2006): "Assortative Matching and Search," Journal of Political Economy, 114(6), 1124-1146. 